\title{
The Ehrenfest Oscillations in The Level Statistics of Chaotic Quantum Dots
}

\author{
Chushun $\operatorname{Tian}^{1}$ and Anatoly I. Larkin ${ }^{1,2}$ \\ 1 William I. Fine Theoretical Physics Institute, University of Minnesota, Minneapolis, MN 55455 \\ ${ }^{2}$ L.D.Landau Institute for Theoretical Physics, Moscow, 117940, Russia
}

(November 8, 2018)

\begin{abstract}
We study a crossover from classical to quantum picture in the electron energy statistics in a system with broken time-reversal symmetry. The perturbative and nonperturbative parts of the two level correlation function, $R(\omega)$ are analyzed. We find that in the intermediate region, $\Delta \ll$ $\omega \sim t_{E}^{-1} \ll t_{\text {erg }}^{-1}$, where $t_{E}$ and $t_{\text {erg }}$ are the Ehrenfest and ergodic times, respectively, $R(\omega)$ consists of a series of oscillations with the periods depending on $t_{E}$, deviating from the universal WignerDyson statistics. These Ehrenfest oscillations have the period dependence as $t_{E}^{-1}$ in the perturbative part. [For systems with time-reversal symmetry, this oscillation in the perturbative part of $R(\omega)$ was studied in an earlier work (I. L. Aleiner and A. I. Larkin, Phys. Rev. E 55, R1243 (1997))]. In the nonperturbative part they have the period dependence as $\left(\Delta^{-1}+\alpha t_{E}\right)^{-1}$ with $\alpha$ a universal numerical factor. The amplitude of the leading order Ehrenfest oscillation in the nonperturbative part is larger than that of the perturbative part.
\end{abstract}

PACS numbers: 73.23.-b, 73.23.Ad, 73.20.Fz, 05.45.Mt

\section{INTRODUCTION}

In the last decade, there has been increasing interest in investigating interference phenomena of electronic motion in ballistic quantum dots ${ }^{1}$. In semiconductor quantum dots the confining potential has the length scale much larger than the Fermi wavelength, and therefore one may expect that electron motion in such systems may be described by methods of classical trajectories. Typically, the classical electron motion inside dots is chaotic. An important question is, what happens to the energy spectrum. It turns out that the energy spectrum of a single electron in chaotic quantum dots is highly sensitive to the parameters of the system (e.g., Fermi momentum, the strength of the applied magnetic field, the impurity configuration, the shape of the boundary of ballistic quantum dots, etc.). Substantial progress has been made by studying the statistics of energy levels. The level statistics has played an important role in the theories of atomic nuclei ${ }^{2,3}$, disordered metals ${ }^{4-6}$, and quantum $\operatorname{chaos}^{7}$. A particularly interesting quantity in the level statistics is the so-called two level correlation function, $R(\omega)$ :

$$
R(\omega)=\Delta^{2}\left\langle\operatorname{Tr}\left(\epsilon+\frac{\omega}{2}-\hat{H}\right) \operatorname{Tr}\left(\epsilon-\frac{\omega}{2}-\hat{H}\right)\right\rangle
$$

where $\Delta$ is the mean level spacing and $\hat{H}$ is the Hamiltonian. $\langle\cdots\rangle$ denotes the average over a wide energy band (for individual quantum dots) or the impurity configuration (for quantum disorders).

In his pioneering work, Dyson divided the Hamiltonian matrices into three classes: Gaussian orthogonal ensemble (GOE), Gaussian unitary ensemble (GUE) and Gaussian sympletic ensemble (GSE) and used the random matrix theory (RMT) to find that $R(\omega)$ follows different universal behavior for these ensembles ${ }^{8,9}$. Gor'kov and Eliashberg were the first to use Wigner-Dyson ensemble to study transport properties in small metallic grains ${ }^{4}$.
For disordered metals, the behavior of $R(\omega)$ is now well understood. For grains in the presence of a lot of quantum (Born) impurities, theoretical justification of the universal RMT result came up with the supersymmetric field theory due to Efetov $^{5}$, assuming that $\omega$ is much smaller than the Thouless energy $E_{T h}=\hbar D / L^{2}$ with $D$ being the diffusion constant and $L$ the size of the grain. The behavior of $R(\omega)$ beyond $E_{T h}$ is nonuniversal ${ }^{6}$. That is, the level statistics at $\omega \gtrsim E_{T h}$ is system-dependent. In Ref. 10, it was found that there is a small constant correction to $R(\omega)$ at $\omega \sim \Delta$ due to the nonuniversal behavior of electron motion. Recently, the weak localization correction to $R(\omega)$ beyond the Thouless energy $E_{T h}$ was studied in Ref. 11 with the help of replica technique ${ }^{12}$.

Bohigas, Giagonni, and Schmit (BGS) proposed a conjecture that the fluctuations of levels of quantum chaotic systems may follow the universal RMT results ${ }^{13}$. One routine method to study the behavior of $R(\omega)$ in ballistic quantum dots is to employ the Gutzwiller formula ${ }^{14}$. A cornerstone was put by Berry ${ }^{15}$. By taking into account the diagonal contributions, he was able to use the Hannay-Ozorio de Almeida (HOA) sum rule ${ }^{16}$ to reproduce the leading perturbative term of the universal RMT results. Proceeding along this line, an important progress was made in Ref. 17, where the off-diagonal contributions were studied and the oscillation similar to the universal RMT results was thereby found.

In this paper, we will study the ballistic quantum dot with weak diffractions, where the classical electronic motion is chaotic (chaotic quantum dots). In such case, there is a new scale $t_{E}$, so-called Ehrenfest time, which has logarithmic dependence on $\hbar$ :

$$
t_{E}=\frac{1}{\lambda}\left|\ln \left(\frac{L}{\lambda_{F}}\right)\right| \sim \frac{1}{\lambda}|\ln \hbar|
$$

Here $\lambda$ is the Lyapunov exponent, $L$ is some macroscopic size and $\lambda_{F}$ is the Fermi wavelength. Physically, 
it is the time scale for an initial Gaussian wave packet (with the typical spatial spreading $\sim \lambda_{F}$ ) to develop to some macroscopic size. At this scale, the electron motion shows a crossover from classical to quantum picture. Such a crossover exists in other phenomena also. Larkin and Ovchinikov ${ }^{18}$ found that it is possible to use the method of classical trajectories in the theory of superconductor for $t<t_{E}$ only. Besides, they were able to estimate $t_{E}$ for Lorentz gases of semiclassical impurities. The logarithmic dependence on $\hbar$ of $t_{E}$ was also found by Berman and Zaslavsky in the studies of classical chaos of the kicked rotator model ${ }^{19}$. In the past two decades, it has been found that the Ehrenfest time plays an important role in condensed matter. Wilkinson addressed the importance of such a scale in the semiclassical studies of sum rules over generic matrix elements ${ }^{20}$. Argaman studied the conductance in the scale $\omega \ll t_{E}^{-121}$. In Ref. 22, Aleiner and Larkin not only gave the estimation for $t_{E}$, but also studied the weak localization correction to the conductivity at the crossover scale $\omega \sim t_{E}^{-1}$. They showed that diffusons do not couple at time $t<t_{E}$ while at time $t \gtrsim t_{E}$, the coupling reaches a universal value. As a result, the amplitude of the coupling between diffusons (Hikami box $)^{23}$ is an oscillating function of $\omega t_{E}$. Later it was found that such amplitude has important applications in studying the crossover from classical and quantum picture of shot noise $\mathrm{e}^{24,25}$ and the density of states (DOS) in Andreev billiard ${ }^{26,27}$.

For chaotic quantum dots with one macroscopic scale $L$, the ergodic time $t_{\text {erg }} \sim L / v_{F}$, which is of the same order of $\lambda^{-1}$. In the semiclassics, $t_{E}$ opens a new region: $\Delta \ll t_{E}^{-1} \sim \omega \ll t_{\text {erg }}^{-1}$. The manifestation of $t_{E}$ in the level statistics of chaotic quantum dots was first studied in Ref. 28. According to the RMT, asymptotically, for large $\omega$ the two level correlation function consists of two parts: One part is perturbative in $\frac{\Delta}{\omega}$. The other is nonperturbative, which oscillates with the period $\Delta$. In Ref. 28, it was found that there is a small oscillation with the period proportional to $t_{E}^{-1}$ correcting the perturbative part of the universal $R(\omega)$ for $\mathrm{GOE}^{29}$ :

$$
\Delta R_{p}^{\mathrm{o}}(\omega)=\frac{\Delta^{3}}{2 \pi^{3}} \operatorname{Re} \frac{\partial^{2}}{\partial(-i \omega)^{2}} \frac{\Gamma_{2}^{2}(\omega)}{-i \omega},
$$

where $\Gamma_{2}(\omega)$ is

$$
\Gamma_{2}(\omega)=\exp \left(i \omega t_{E}-\frac{\omega^{2} \lambda_{2} t_{E}}{\lambda^{2}}\right) .
$$

Here $\lambda$ is the Lyapunov exponent and $\lambda_{2}$ characterizes the fluctuations of $\lambda$ [see Eqs. (A15) and (A16) for the general definitions]. Typically, $\lambda$ and $\lambda_{2}$ are of the same order.

In this paper, we study the two level correlation function in the system with broken time-reversal symmetry. We recall that in this case, the RMT predicts the famous result that the two level correlation function is described by the elegant formula:

$$
R^{\mathrm{u}}(\omega)=1-\frac{\Delta^{2}}{2 \pi^{2} \omega^{2}}\left(1-\cos \frac{2 \pi \omega}{\Delta}\right)
$$

Asymptotically, for large $\omega$, the two level correlation function is exactly truncated at the $(\Delta / \omega)^{2} \operatorname{term}^{5,8}$. An important question is whether it is the property of generic systems with broken time-reversal symmetry or just comes out with the RMT for GUE. In this paper, we will show that Eq. (5) just comes out with RMT, or describes the universal limit of the two level correlation function in quantum disorders. For ballistic quantum dots, surprisingly, we find that in the crossover region $t_{E}^{-1} \sim \omega \ll t_{\text {erg }}^{-1}$, the behavior of the two level correlation function deviates from the universal Wigner-Dyson statistics given by Eq. (5). More exactly, such deviations are formulated as

$$
\Delta R^{\mathrm{u}}(\omega)=\Delta R_{p}^{\mathrm{u}}(\omega)+\Delta R_{n p}^{\mathrm{u}}(\omega)
$$

where

$$
\Delta R_{p}^{\mathrm{u}}(\omega)=-\frac{\Delta^{4}}{8 \pi^{4}} \operatorname{Re} \frac{\partial^{2}}{\partial(-i \omega)^{2}}\left[\frac{\Gamma_{2}^{4}(\omega)}{(-i \omega)^{2}}-\frac{\Gamma_{3}^{2}(\omega)}{(-i \omega)^{2}}\right],
$$

and

$$
\Gamma_{3}(\omega)=\exp \left(\frac{3}{2} i \omega t_{E}-\frac{9}{4} \frac{\omega^{2} \lambda_{2} t_{E}}{\lambda^{2}}\right)
$$

$$
\Delta R_{n p}^{\mathrm{u}}(\omega)=2 \frac{\Delta^{3}}{\pi^{3} \omega^{3}} \sin \frac{2 \pi \omega}{\Delta} \operatorname{Re}\left[\Gamma_{2}^{2}(\omega)-\omega \frac{\partial}{\partial \omega} \Gamma_{2}^{2}\left(\frac{\omega}{2}\right)-1\right] .
$$

Compared to Eq. (3), the deviations in systems with broken time-reversal symmetry are more interesting. First, we point out that the main feature of these deviations is the appearance of two types of oscillations in addition to the oscillation described by the WignerDyson statistics. One type correcting the perturbative terms has the period $\sim t_{E}^{-1}$, which has the similar origin as Eq. (3). In fact, as we will show in Sec. III, Eq. (3) comes from the two loop approximation. In the broken time-reversal system, the two loop approximation vanishes. Instead, Eq. (7) comes from the three loop approximation. They are the weak localization corrections to leading universal $R(\omega)$ in the crossover region. Eq. (9) belongs to the other type of oscillation, which corrects the nonperturbative part of the universal $R(\omega)$ described by the universal Wigner-Dyson statistics and was not found in the previous work ${ }^{28}$. It has the period $\left(\Delta^{-1}+\alpha t_{E}\right)^{-1}$. Both of them have $t_{E}$-dependent periods. In this way, we term them the Ehrenfest oscillations. In the broken time-reversal system, the oscillation with the period $\left(\Delta^{-1}+\alpha t_{E}\right)^{-1}$ is stronger than the oscillation with period $\sim t_{E}^{-1}$. For the time-reversal system, the Ehrenfest oscillation with the period $\left(\Delta^{-1}+\alpha t_{E}\right)^{-1}$ 
does exist, but the amplitude is smaller than that described by the Wigner-Dyson statistics, which is even small.

In Ref. 30, it is proposed that the amplitudes of both perturbative and nonperturbative parts of $R(\omega)$ are related to each other through the classical spectrum determinant, which depends on the eigenvalues of the PerronFrobenius operator. In the universal limit $\omega \ll t_{\text {erg }}^{-1}$, it coincides with the result of RMT. Indeed, for the broken time-reversal system, we see from Eq. (5) that the amplitudes of both parts are in the same order. However, such a conjecture does not exist once the quantum corrections are taken into account. In fact, the leading quantum correction in the perturbative part, i.e., Eq. (7) comes from the three loop approximation, while the leading quantum correction in the nonperturbative part, i.e., Eq. (9) comes from the two-loop approximation. As a result, the leading order quantum corrections lead to the Ehrenfest oscillations with different power in $\Delta / \omega$.

It is worth pointing out that the methods of calculating coupled multi-diffusons (see Sec. IV) involved in the three loop approximation are general. The idea may be applied to find the weak localization correction to other physical quantities (e.g. conductivity) in the ballistic quantum dots.

Technically, it is convenient to use the ballistic nonlinear supermatrix $\sigma$ model $(\mathrm{BNS} \sigma \mathrm{M})^{30-32}$. We will introduce this model in the next section. The quantum transport time, essential to the regularizer will be estimated. The supersymmetric action has two saddle points: $\Lambda$ and $-k \Lambda^{33}$. The perturbative expansion around $\Lambda$ gives the weak localization corrections to the leading perturbative term in the Wigner-Dyson statistics, while the perturbative expansion around $-k \Lambda$ gives the quantum corrections to the leading Wigner-Dyson oscillation. In Sec. III, we consider the perturbative $R(\omega)$ for systems with timereversal symmetry. The perturbation theory near $\Lambda$ will be developed. In Sec. IV, we consider systems with broken time-reversal symmetry. The perturbation theory near $\Lambda$ will be employed to study the weak localization corrections in $R_{p}(\omega)$. The products of the perturbative expansion involve the product of multi-diffusons. We will calculate such coupled diffusons. Immediately, the leading order quantum corrections give the Ehrenfest oscillations in $R_{p}^{\mathrm{u}}(\omega)$. In Sec. V, with the help of the global transformation, we will perform the perturbation expansion near $-k \Lambda$. Consequently, we find the Ehrenfest oscillations in the nonperturbative part of $R(\omega)$ but with different dependence of the period on $t_{E}$. The obtained results are summarized in Sec. VI. Some of the calculations of multi-diffusons are included in Appendix A.

\section{BALLISTIC NONLINEAR SUPERMATRIX $\sigma$ MODEL (BNS $\sigma \mathrm{M})$}

Diffusive nonlinear supermatrix $\sigma$ model has become a powerful tool in the studies of $R(\omega)$ in quantum disorders ${ }^{5,33}$. A natural question is where such a technique can be generalized to the ballistic case, especially individual quantum dots, whether the average over impurity configurations become impossible. Towards this direction, the first suggestion is given in Ref. 31, where a ballistic action was phenomenologically proposed. The formal justification is obtained in Ref. 30 under some crucial assumptions ${ }^{34}$. The complete microscopic derivation is given for long-ranged disorders ${ }^{32,35}$. In this section, we will introduce BNS $\sigma$ M. Moreover, the roles played by the regularizer will be discussed.

\section{A. Ballistic supersymmetric action}

It is a standard method to introduce a supermatrix field $Q$. It is defined on the superspace $p \otimes g \otimes d$, where $p$ stands for the advanced/retarded block, $g$ stands for the fermion/boson block and $d$ stands for the time-reversal block. Similarly to the quantum disordered case ${ }^{5}, R(\omega)$, Eq. (1) can be expressed as the integral over $Q$ :

$$
\begin{aligned}
& R(\omega)=\operatorname{Re} \tilde{R}(\omega), \\
& \tilde{R}(\omega)=\frac{1}{64} \int D Q\left(\int d x_{\|} \operatorname{STr} k \Lambda Q\left(x_{\|}\right)\right)^{2} e^{-S}
\end{aligned}
$$

Here the subscript in $x_{\|}$means that the integration is restricted on the energy shell. $\Lambda$ is a supermatrix defined as:

$$
\Lambda=\left(\begin{array}{cc}
1 & 0 \\
0 & -1
\end{array}\right)_{p} \otimes 1_{g} \otimes 1_{d}
$$

The ballistic supersymmetric action $S$ is:

$$
S=\frac{\pi \nu}{2} \int d 1 \mathrm{~S} \operatorname{Tr}\left[\frac{i \omega^{+}}{2} \Lambda Q-T^{-1} \Lambda \hat{\mathcal{L}} T+\frac{1}{\tau_{q}}\left(\frac{\partial Q}{\partial \phi}\right)^{2}\right]
$$

where $\omega^{+}=\omega+i 0^{+}$with $0^{+}$being an arbitrarily small positive number. $1=(\mathbf{r}, \mathbf{n}), d 1=d \mathbf{r} d \mathbf{n} / 2 \pi . \quad \hat{\mathcal{L}}=$ $\mathbf{v} \cdot \nabla-\nabla U(\mathbf{r}) \cdot \frac{\partial}{\partial \mathbf{p}}$ is the Liouville operator with $U(\mathbf{r})$ being the classical potential. The matrix $Q$ is generated by $T$ :

$$
Q=T^{-1} \Lambda T
$$

and takes the value on the corresponding symmetry space $\mathbf{H}=\mathbf{G} / \mathbf{K}$, where $\mathbf{G}$ and $\mathbf{K}$ are groups. For GUE, $\mathbf{H}=\mathbf{U}(1,1 / 2) / \mathbf{U}(1 / 1) \otimes \mathbf{U}(1 / 1)$. For GOE, $\mathbf{H}=\mathbf{U}(2,2 / 4) / \mathbf{U}(2 / 2) \otimes \mathbf{U}(2 / 2) . T(1)$ satisfies:

$$
T^{\dagger}(1) L T(1)=L,
$$

where $L$ is a matrix defined as

$$
L=\left(\begin{array}{ll}
1 & 0 \\
0 & k
\end{array}\right)_{p} \otimes 1_{d}, \quad k=\left(\begin{array}{cc}
1 & 0 \\
0 & -1
\end{array}\right)_{g} .
$$


The form of the regularizer is determined by the properties of the Liouville operator and diffraction mechanisms for particular systems. In the presence of quantum impurities with small-angle scattering, the regularizer has the form presented in Eq. (12) with $1 / \tau_{q}$ proportional to the impurity density ${ }^{22,28}$. In this paper, we will use it to regularize the Liouville operator. According to the geometric theory of diffractions ${ }^{36}$, when an electron glances off a hard disk with the radius $\rho$, it emits diffraction rays into the shadowed region deviating from the incident ray at the angle $\theta \sim\left(\frac{\lambda_{F}}{\rho}\right)^{2 / 3} 37$. This leads to the estimation: $\frac{1}{\tau_{q}} \sim \frac{v_{F}}{L}\left(\frac{\lambda_{F}}{\rho}\right)^{2 / 3}$, which coincides with the estimation in Ref. 38. For smooth potentials, it is estimated that $\tau_{q}^{-1} \sim v_{F} \lambda_{F} / L^{2} 18,22$, which is also obtained in Ref. 26 by analyzing the spreading of the wave packet. In any cases, $\tau_{q} \sim \hbar^{\alpha}$. It is important that the regularizer is not zero but small. In Ref. 22 (see also Sec. II D), it was shown that the Ehrenfest time $t_{E}=\frac{1}{\lambda} \ln \lambda \tau_{q}$. For $\lambda \tau_{q} \gg 1, t_{E}$ depends on $\tau_{q}$ logarithmically. In the semiclassics, $\tau_{q}$ is large but not infinite. Any change of the form of the regularizer accounts only for the value of the parameter $\tau_{q}$ under logarithm.

\section{B. The perturbation theory near $\Lambda$}

To integrate over all the modes in Eqs. (10) and (12), we will use the saddle point approximation. In quantum disorders, the Gaussian approximation leads to Altshuler and Shklovskii's result ${ }^{33}$. For $\omega<t_{\text {erg }}^{-1}$, only the zero

$$
\tau_{0}=\left(\begin{array}{ll}
1 & 0 \\
0 & 1
\end{array}\right)_{d}, \quad \tau_{1}=\left(\begin{array}{ll}
0 & 1 \\
1 & 0
\end{array}\right)_{d}
$$

act on the time-reversal block. Under the parameterization Eq. (16), we have the following expansion:

$$
\begin{aligned}
\mathrm{STr}[k \Lambda Q]=2 \sum_{j=1}^{\infty} \mathrm{S} \operatorname{Tr}\left[k(-1)^{j} P^{2 j}\right], & \quad(21) \quad \text { and } \\
S & =S^{0}+S^{i n t .}, \\
S^{0} & =\frac{\pi \nu}{2} \int d x_{\|} \mathrm{S} \operatorname{Tr}\left[P\left(-i \omega^{+}+\Lambda \hat{\mathcal{L}}_{R}\right) P\right], \\
S^{\text {int. }} & =-\frac{\pi \nu}{2} \int d x_{\|} \sum_{j=1}^{\infty} \mathrm{STr}\left[(-1)^{j+1} P^{2 j+1}\left(-i \omega^{+}+\Lambda \hat{\mathcal{L}}_{R}\right) P\right] .
\end{aligned}
$$

mode is important, which is coordinate independent. The coupling between diffusive modes accounts for the higher order term in the saddle point approximation. Similarly, in chaotic quantum dots, the the eigenmodes of the regularized Liouville operator may contribute to $R(\omega)$ independently in the Gaussian approximation. Moreover, there is coupling between these eigenmodes also.

The action, Eq. (12) has two saddle points: $\Lambda$ and $-k \Lambda^{33}$. In Sec. III and IV, we will study the case with $Q$ close to $\Lambda$. As in the disordered case, $R_{p}(\omega)$ is dominated by this saddle point ${ }^{30,33}$. It is convenient to choose the parameterization below for $T$ :

$$
T=1+i P
$$

The Jacobian of this transformation is unity. $P$ anticommutes with $\Lambda$ :

$$
\Lambda P+P \Lambda=0, \quad P=\left(\begin{array}{cc}
0 & B \\
\bar{B} & 0
\end{array}\right) .
$$

According to Eq. (14), $P(1)$ satisfies the following condition:

$$
P(1)^{*}=-C P(\overline{1}) C^{T}
$$

where $\overline{1}=(\mathbf{r},-\mathbf{n})$ and $C$ is the charge conjugation matrix:

$$
C=1_{p} \otimes\left(\begin{array}{cc}
-i \tau_{2} & 0 \\
0 & \tau_{1}
\end{array}\right)_{g}
$$

The Pauli matrices:
Here the Perron-Frobenius operator $\hat{\mathcal{L}}_{R}$ is defined as ${ }^{39}$ :

$$
\hat{\mathcal{L}}_{R}=\mathbf{v} \cdot \nabla-\nabla U(\mathbf{r}) \cdot \frac{\partial}{\partial \mathbf{p}}-\frac{1}{\tau_{q}} \frac{\partial^{2}}{\partial \phi^{2}}
$$

These expansions are essential to the perturbation calcu- lations.

Keeping the terms up to $P^{2}$, we reproduce the leading terms of the universal Wigner-Dyson results for $R_{p}(\omega)$ [see Eqs. (27) and (28)]. In Sec. III and IV, we will calculate the weak localization correction to $R_{p}(\omega)$. The 
feature of finite $t_{\text {erg }}$ implies the existence of the gap in the spectrum of Perron-Frobenius operator, which has been found in many chaotic systems ${ }^{40,41}$. we will drop out the $P^{2 j+1} \Lambda \hat{\mathcal{L}}_{R} P$ term in the effective interaction $S^{i n t}$. hereafter since it gives much smaller contribution at $\omega \ll t_{\text {erg }}^{-1}$.

\section{Quantum disorders}

Now we discuss the $\tau_{q}$ dependence of $R(\omega)$. In the presence of many quantum impurities (i.e., quantum disorders), $\tau_{q}$ becomes very small $\left(v_{F} / L \ll 1 / \tau_{q}\right)$. The last term in the action suppresses all the nonzero modes. In this case, one can drop out the last two terms in Eq. (12) and put $Q$ to be a constant matrix ${ }^{5}$. In this way, the universal RMT results are reproduced. For GOE,

$$
\begin{aligned}
R^{\mathrm{o}}(\omega) & =1-\frac{\Delta^{4}}{\pi^{4} \omega^{2}} \sin ^{2} \frac{\pi \omega}{\Delta} \frac{d}{d \omega}\left[\frac{1}{\omega} \sin \frac{\pi \omega}{\Delta}\right] \int_{1}^{\infty} \frac{1}{t} \sin \frac{\pi \omega t}{\Delta} d t \\
& \simeq 1-\frac{\Delta^{2}}{\pi^{2} \omega^{2}}-\frac{\Delta^{4}}{\pi^{4} \omega^{4}}\left(\frac{3}{2}+\frac{1}{2} \cos \frac{2 \pi \omega}{\Delta}\right)
\end{aligned}
$$

For GUE,

$$
R^{\mathrm{u}}(\omega)=1-\frac{\Delta^{2}}{\pi^{2} \omega^{2}} \sin ^{2} \frac{\pi \omega}{\Delta}=1-\frac{\Delta^{2}}{2 \pi^{2} \omega^{2}}\left(1-\cos \frac{2 \pi \omega}{\Delta}\right)
$$

In the second line of Eq. (27), we take the limit $\omega \gg \Delta$. Actually, for large $\omega R(\omega)$ can be asymptotically expressed as

$$
\begin{gathered}
R(\omega)=R_{p}(\omega)+R_{n p}(\omega) \\
R_{p}(\omega)=1+\sum_{n}\left(\frac{\Delta}{\omega}\right)^{n} C_{n} \\
R_{n p}(\omega)=\cos \frac{2 \pi \omega}{\Delta} \sum_{n}\left(\frac{\Delta}{\omega}\right)^{n} D_{n}+\sin \frac{2 \pi \omega}{\Delta} \sum_{n}\left(\frac{\Delta}{\omega}\right)^{n} E_{n}
\end{gathered}
$$

where $C_{n}, D_{n}$ and $E_{n}$ are universal numerical constants. $R(\omega)$ consists of two parts: One [denoted by $R_{p}(\omega)$ ] is perturbative in $\Delta / \omega$ and the other [denoted by $R_{n p}(\omega)$ ] is nonperturbative. It is important that expanding $R_{p}(\omega)$ in terms of $\Delta / \omega$ is a result of making the saddle point approximation to Eqs. (10) and (12) (see Sec. III). That is, we expand $Q$ near $\Lambda$ (i.e., $T$ near 1) and take into account the fluctuations of $T$ perturbatively. In particular, terms like $(\Delta / \omega)^{n}, n>2$ come from perturbative corrections to the Gaussian approximation in Eq. (10). Actually in the zero mode nonlinear $\sigma$ model, the action depends only on the parameter $\omega / \Delta$. As a result, the expansion of $R(\omega)$, Eqs. (29)-(31) has no other parameter dependence. The reason is that in the disordered case, the Hikami box ${ }^{23}$ of zero modes has dispersion only at $\omega \sim \tau_{t r}^{-1} \gg E_{T h}$, which are unimportant in the universal region $\omega \ll E_{T h}$. For $\omega \gtrsim E_{T h}$, nonzero diffusive modes are important and $R_{p}(\omega)$ becomes nonuniversal ${ }^{6}$. In the disordered case, the weak localization correction to nonuniversal $R_{p}(\omega)^{6}$ is studied in Ref. 11 .

\section{The Ehrenfest time $t_{E}$}

As in the diffusive case ${ }^{5}$, one may try to use the zero mode only and thereby drop out the free Liouville term as well as the regularizer in Eq. (10) in the region $\omega \ll t_{\text {erg }}^{-1}$ to recover universal RMT results. However, this procedure may miss important physics as we will present in this paper. To favor uniform $Q$, a necessary condition is that the regularizer becomes comparable to the Liouville term, which is of the order of $\sim v_{F} / L$. As the initial nonuniform $Q$ relaxes to the zero mode, the regularizer gets increasing $\sim \frac{1}{\tau_{q}} e^{\lambda t}$ due to the Lyapunov instability. The two terms become comparable at $t \sim t_{E}=\lambda^{-1}\left|\ln \left(v_{F} \tau_{q} / L\right)\right|$. Hence we conclude that only in the region $\omega \ll t_{E}^{-1}, Q$ can be put to be a constant matrix in the supersymmetric action to reproduce the universal RMT results.

It is important that as usual, in the region $\omega \ll t_{E}^{-1}$, the diffusons become self-averaging in an individual quantum dot. The reason is that the time scale for the deterministic chaos to become random is $t_{\text {erg }}$. However, the time for two identical trajectories (up to the Heisenberg un- 
certainty) to decouple (i.e., become independent of each other) is much longer, as we will show in Sec. V, is of the order of $t_{E}$. Thus diffusons with the same coordinates are strongly coupled in the region $t_{E}^{-1} \sim \omega \ll t_{\text {erg }}^{-1}$. The Hikami box thereby acquires an additional dispersion at $\omega \sim t_{E}^{-1}$ and crossovers to the disordered limit at $\omega \ll t_{E}^{-1}$. As we mentioned earlier, for GOE, the appearance of $(\Delta / \omega)^{4}$ term is a pure quantum effect. Indeed, it comes from the coupling between two zero mode diffusons. More strictly speaking, it comes from the coupling between a diffuson and a cooperon through Hikami box. In this way, it is appropriate to identify such a term as the weak localization correction to $R_{p}(\omega)$. For GUE, the exact truncation at $(\Delta / \omega)^{2}$ term by no means implies the invalidity of the perturbation theory, but is due to the exact cancellation of the weak localization correction to $R_{p}(\omega)$ arising from Hikami boxes with 4-point and 6point vertex, respectively. In Sec. IV C and $\mathrm{VC}$, we will see that the $(\Delta / \omega)^{4}$ term shows up associating with the Ehrenfest oscillation in chaotic quantum dots.

In this paper, we will study $R(\omega)$ in the region $\Delta \ll$ $\omega \ll t_{\text {erg }}^{-1}$. For typical quantum dots, there is only one macroscopic scale $L$. The Lyapunov exponent $\lambda$ is of the same order of $v_{F} / L \sim t_{\text {erg }}^{-1}$. Thus in the crossover from semiclassical to quantum picture, the Ehrenfest time effectively opens a new region $\Delta \ll t_{E}^{-1} \sim \omega \ll t_{\text {erg }}^{-1}$.

\section{E. Classical limit $\tau_{q} \rightarrow \infty$}

It is important that the regularizer introduces couplings between different primitive periodic orbits. In the absence of diffractions or quantum impurities, $\tau_{q}$ goes to the infinity. In such a limit, Kogan and Efetov proved that Eqs. (10) and (12) are compatible with the Gutzwiller trace formula ${ }^{32}$. Furthermore one may apply the HOA sum rule to reproduce the leading perturbative term in $R_{p}(\omega)$, which is of the order of $1 / \omega^{2}$ [see Eqs. (27) and (28)]. It is important that the constant term 1 (so-called Weyl term) in $R_{p}(\omega)$, is pure classical which characterizes the phase space volume of the energy shell ${ }^{7}$. So is the $\omega^{-2}$ term, which arises from the interference between two identical primitive periodic classical orbits (diagonal contribution) $)^{15,17}$. Recently, by taking into account the off-diagonal contributions, an oscillation similar to those in Eqs. (27) and (28) was found ${ }^{17}$.

Thus, we conclude that in the classical limit $\tau_{q} \rightarrow \infty$, Eqs. (10) and (12) give the leading perturbative and nonperturbative terms. We obtain the same results by making the Gaussian approximation to Eqs. (10) and (12). This is not surprising. We argue that to pass from the Gutzwiller formula to the leading $1 / \omega^{2}$ term, the only condition is the ergodicity, hence it is reasonable to expect that the saddle point approximation remains good enough in the region $\Delta \ll \omega \ll t_{\text {erg }}^{-1}$. Actually, such a term results from the free motion of diffusons. It is worth noticing that so far the next to leading term $\sim \frac{1}{\omega^{4}}$ in $R_{p}(\omega)$, as one may expect from the universal RMT results [see Eq. (27)], has not yet been identified in the framework of Gutzwiller formula. As we discussed in Sec. IID, it is a pure quantum effect (weak localization correction).

\section{THE PERTURBATIVE $R(\omega)$ IN SYSTEMS WITH TIME-REVERSAL SYMMETRY}

For systems with time-reversal symmetry, the weak localization correction to $R_{p}(\omega)$ in chaotic quantum dots was first studied by Aleiner and Larkin ${ }^{28}$. Furthermore, we show in this subsection that such a correction can be written as the second derivative of the free function at the two loop levels. This is important because it suggests that the result here, in principle, is possible to be reproduced by using the replica technique ${ }^{11,42}$.

\section{A. Weak localization corrections to the perturbative $R(\omega)$}

From Eqs. (10), (21) and (25), we can find that $R_{p}(\omega)$ in the order of $(\Delta / \omega)^{3}$ is

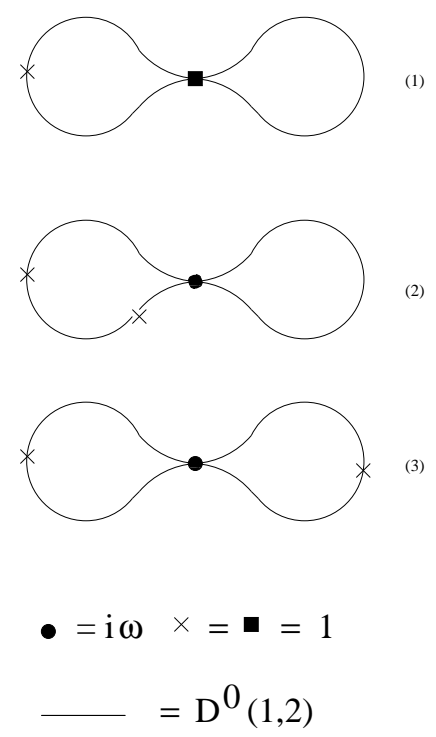

FIG. 1. The diagrams contribute to $R_{p}(\omega)$ in the order of $(\Delta / \omega)^{3}$ in systems with time-reversal symmetry. The cross and the square, $\sim \operatorname{Str}\left[k P^{2}\right]$ and $\sim \operatorname{Str}\left[k P^{4}\right]$, respectively, come from the expansion in the prefactor, see Eq. (21). The $\operatorname{dot}, \sim i \omega \operatorname{Str}\left[P^{4}\right]$, comes from the effective interaction, see Eq. (25). In the figure, we put $\Delta=1$.

$$
\Delta R_{p}^{\mathrm{o}}(\omega)=-\frac{1}{8} \operatorname{Re} \int D P e^{-S^{0}} \int d 1 \mathrm{~S} \operatorname{Tr}\left[k P^{4}(1)\right] \int d 2 \mathrm{~S} \operatorname{Tr}\left[k P^{2}(2)\right]-
$$




$$
\frac{1}{16} \operatorname{Re} \int D P e^{-S^{0}} \int d 1 \mathrm{~S} \operatorname{Tr}\left[k P^{2}(1)\right] \int d 2 \mathrm{~S} \operatorname{Tr}\left[k P^{2}(2)\right] \cdot\left(\frac{1}{2} i \pi \nu \omega\right) \int d 3 \mathrm{~S} \operatorname{Tr}\left[P^{4}(3)\right],
$$

where we keep the term with $j=1$ in the effective interaction $S^{\text {int }}$.

To calculate Eq. (32), we need to use the Wick's theorem, which implies that any even order moments of the Gaussian integral can be factorized as the product of the moment $P^{2}$ of the Gaussian integral. The contraction rules $\operatorname{are}^{28}$ :

$$
2 \pi \nu \overbrace{P(1) M} P(2)=\mathcal{D}^{0}(\overline{1}, \overline{2}) \Lambda_{\|}^{+} \operatorname{STr}\left[M \Lambda_{\|}^{-}\right]+\mathcal{D}^{0}(1,2) \Lambda_{\|}^{-} \operatorname{STr}\left[M \Lambda_{\|}^{+}\right]+\mathcal{D}^{0}(1, \overline{2}) \Lambda_{\|}^{-} \bar{M} \Lambda_{\|}^{+}+\mathcal{D}^{0}(\overline{1}, 2) \Lambda_{\|}^{+} \bar{M} \Lambda_{\|}^{-}
$$

and

$$
2 \pi \nu \mathrm{S} \operatorname{Tr}[M P \overbrace{(1)] \operatorname{STr}[N} P(2)]=\operatorname{S} \operatorname{Tr}\left[\left(\mathcal{D}^{0}(1,2) M-\mathcal{D}^{0}(\overline{1}, 2) \bar{M}\right) \Lambda_{\|}^{-} N \Lambda_{\|}^{+}+\left(\mathcal{D}^{0}(\overline{1}, \overline{2}) M-\mathcal{D}^{0}(1, \overline{2}) \bar{M}\right) \Lambda_{\|}^{+} N \Lambda_{\|}^{-}\right] .
$$

Here $\Lambda_{\|}^{ \pm}=\frac{1}{2}(1 \pm \Lambda)$ and $\bar{M}=K C M^{T} C^{T} K$. The diffuson $\mathcal{D}^{0}(1,2)$ is the solution of the regularized Liouville (Perron-Frobenius) equation:

$$
\left(-i \omega+\hat{\mathcal{L}}_{R, 1}\right) \mathcal{D}^{0}(1,2)=2 \pi \delta(1-2) .
$$

$\mathcal{D}^{0}(1,2 ; t)$ is the conditional probability for the particle initially at 2 to appear at 1 . At the limit $\tau_{q} \rightarrow \infty, \mathcal{D}^{0}(1,2 ; t)=$ $\delta\left(\mathbf{R}_{1}-\mathbf{R}_{2}(t)\right) \delta\left(\mathbf{n}_{1}-\mathbf{n}_{2}(t)\right)$ with $\left(\mathbf{R}_{2}(t), \mathbf{n}_{2}(t)\right)$ the classical trajectory starting from $\left(\mathbf{R}_{2}(0), \mathbf{n}_{2}(0)\right)=\left(\mathbf{R}_{2}, \mathbf{n}_{2}\right)$.

By using Eqs. (33), (34) and the following relation:

$$
\frac{\partial}{\partial(i \omega)} \mathcal{D}^{0}(1,3)=\int d 2 \mathcal{D}^{0}(1,2) \mathcal{D}^{0}(2,3),
$$

we can calculate the first term in Eq. (32) as

$$
\begin{aligned}
I_{1} & =2\left(\frac{\Delta}{\pi}\right)^{3} \int d 1 d 2\left\langle\mathcal{D}^{0}(1,2) \mathcal{D}^{0}(2, \overline{1}) \mathcal{D}^{0}(\overline{1}, 1)\right\rangle \\
& =-2\left(\frac{\Delta}{\pi}\right)^{3} \int d 1\left\langle\left[\frac{\partial}{\partial(-i \omega)} \mathcal{D}^{0}(1, \overline{1})\right] \mathcal{D}^{0}(\overline{1}, 1)\right\rangle .
\end{aligned}
$$

This gives the contraction shown in Fig. 1 (1). Then using the contraction rules, Eqs. (33) and (34) we can find the second term consists of two parts:

$$
\begin{aligned}
I_{2} & =2\left(\frac{\Delta}{\pi}\right)^{3} i \omega \int d 1 d 2 d 3\left\langle\mathcal{D}^{0}(1,2) \mathcal{D}^{0}(2,3) \mathcal{D}^{0}(\overline{3}, 1) \mathcal{D}^{0}(3, \overline{3})\right\rangle \\
& =-\left(\frac{\Delta}{\pi}\right)^{3}(-i \omega) \int d 3\left\langle\left[\frac{\partial^{2}}{\partial(-i \omega)^{2}} \mathcal{D}^{0}(\overline{3}, 3)\right] \mathcal{D}^{0}(3, \overline{3})\right\rangle,
\end{aligned}
$$

and

$$
\begin{aligned}
I_{3} & =-\left(\frac{\Delta}{\pi}\right)^{3}(-i \omega) \int d 1 d 2 d 3\left\langle\mathcal{D}^{0}(\overline{3}, 1) \mathcal{D}^{0}(1,3) \mathcal{D}^{0}(3,2) \mathcal{D}^{0}(2, \overline{3})\right\rangle \\
& =-\left(\frac{\Delta}{\pi}\right)^{3}(-i \omega) \int d 3\left\langle\left[\frac{\partial}{\partial(-i \omega)} \mathcal{D}^{0}(\overline{3}, 3)\right]\left[\frac{\partial}{\partial(-i \omega)} \mathcal{D}^{0}(3, \overline{3})\right]\right\rangle
\end{aligned}
$$

These correspond to the contractions, (2) and (3) in Fig. 1, respectively. Here $\langle\cdots\rangle$ denotes the self-averaging over the energy spectrum. Adding them together, we find that Eq. (32) gives the weak localization correction to $R_{p}(\omega)$ as

$$
\Delta R_{p}^{\mathrm{o}}(\omega)=\left(\frac{\Delta}{\pi}\right)^{2} \operatorname{Re} \frac{\partial^{2}}{\partial(-i \omega)^{2}} F_{2}(\omega)
$$

where the free energy $F_{2}(\omega)$ is

$$
F_{2}(\omega)=i \omega \int d 1\left\langle\mathcal{D}^{0}(1, \overline{1}) \mathcal{D}^{0}(\overline{1}, 1)\right\rangle .
$$


Actually in the r.h.s. of Eq. (41), one $\mathcal{D}^{0}$ comes from the diffuson and the other $\mathcal{D}^{0}$ comes from the cooperon ${ }^{43}$.

$F_{2}(\omega)$ is the ballistic counterpart of the free energy in disordered systems, which is obtained by using the replica technique ${ }^{11}$. In the disordered case or in the limit $\Delta \ll \omega \ll t_{E}^{-1}$, one can justify that $F_{2}(\omega)$ is imaginary by substituting zero mode diffuson, i.e. $\mathcal{D}^{0}(1,2)=1 /-i \omega$ into Eq. (41). As a result, according to Eq. (40), $\Delta R_{p}^{o}(\omega)$ vanishes.

\section{B. The Ehrenfest oscillations}

Now we show that for chaotic quantum dots, in the crossover region $t_{E}^{-1} \sim \omega \ll t_{\text {erg }}^{-1}$, Eqs. (40) and (41) lead to the Ehrenfest oscillations with the period $\sim t_{E}^{-1}$.

We note that the diffusons $\mathcal{D}^{0}(1, \overline{1})$ and $\mathcal{D}^{0}(\overline{1}, 1)$ are decoupled, which means

$$
\left\langle\mathcal{D}^{0}(1, \overline{1}) \mathcal{D}^{0}(\overline{1}, 1)\right\rangle=\left\langle\mathcal{D}^{0}(1, \overline{1})\right\rangle\left\langle\mathcal{D}^{0}(\overline{1}, 1)\right\rangle .
$$

Actually $\mathcal{D}^{0}(1, \overline{1})$ stands for the probability of a trajectory initiating from 1 and returning to $\overline{1}$ in the phase space, while $\mathcal{D}^{0}(\overline{1}, 1)$ stands for the probability of a trajectory initiating from $\overline{1}$ and returning to 1 . Such two trajectories start from the same place in the real space with opposite directions. In this way, they are distant parts in the phase space. Therefore, we have Eq. (42).

In Ref. 22, it was found that

$$
\mathcal{D}^{0}(1, \overline{1})=\frac{\Gamma_{2}(\omega)}{-i \omega^{+}} .
$$

As a result,

$$
\left\langle\mathcal{D}^{0}(1, \overline{1}) \mathcal{D}^{0}(\overline{1}, 1)\right\rangle=\frac{\Gamma_{2}^{2}(\omega)}{(-i \omega)^{2}} .
$$

From this we see that $\Gamma_{2}^{2}(\omega)$ is the effective Hikami box. In the limit $\omega \ll t_{E}^{-1}, \Gamma_{2}^{2}(\omega) \rightarrow 1$. The Hikami box becomes a constant, as in the diffusive disordered case ${ }^{23}$. In the crossover region, it acquires an additional dispersion at $\omega \sim t_{E}^{-1}$.

Substituting Eqs. (42) and (44) into Eq. (40), we obtain Eq. $(3)^{28,29}$. Thus $R_{p}^{\mathrm{o}}$ acquires an Ehrenfest oscillation correction with the period $\sim t_{E}^{-1}$. Such an oscillation originates from the coupling between a diffuson and a cooperon, which is the reminiscence of Hikami box in the quantum disorders. In this way, essentially it is different from the Wigner-Dyson oscillation In the low $\omega$ limit $\Delta \sim \omega \ll t_{E}^{-1}$, such an oscillation gradually diminishes. Actually similar corrections to $R_{p}(\omega)$ persist in higher order terms.

Alternatively, one may Fourier transform $R(\omega)$ as the form factor $K(t)=\frac{\pi}{\Delta} \int \tilde{R}^{o}(\omega) e^{-i \frac{\pi \omega}{\Delta} t} d \omega$ before taking the real part. $R(\omega)=\operatorname{Re} \tilde{R}^{\mathrm{o}}(\omega)$ [cf. Eq. (3)]. The RMT pre$\operatorname{dicts} K(t) \simeq 2 t-t^{2}$ at $t \ll 1^{5,8}$. We know that the linear term is pure classical (see Sec. IIE). In fact, from Eq. (3) we see that $K(t)=2 t-t^{2} \theta\left(t-t_{E}\right)$ at $\lambda_{2}=0$. At $t<t_{E}$ the $t^{2}$ term disappears, while at $t>t_{E}$ it coincides with that predicted by the zero dimensional nonlinear $\sigma$ model $^{5}$. In Ref. 44, it is proved that in the case of $\lambda_{2}=0$, classical trajectories do not contribute to the $-t^{2}$ term even if the off-diagonal contributions are considered. To reproduce this term, the authors of Ref. 44 introduce some correction to the probability of self-crossing classical trajectories.

In this section, we prove the existence of the free energy at the two loop level by using BNS $\sigma \mathrm{M}$ [see Eqs. (40), (41) and (3)]. In fact, as we will see in the next section, at the three-loop level, the free energy does exist also. In this way, it is natural to expect that in general, we can write the perturbative part of $R(\omega)$ as the second order derivative of the free energy $F(\omega)$ i.e.,

$$
R_{p}(\omega)=\left(\frac{\Delta}{\pi}\right)^{2} \operatorname{Re} \frac{\partial^{2}}{\partial(-i \omega)^{2}} F(\omega)
$$

Eq. (45) was first established for quantum disorders by using the replica technique ${ }^{11}$. We are not aware of any proof about it in quantum chaos.

\section{THE PERTURBATIVE $R(\omega)$ IN SYSTEMS WITH BROKEN TIME-REVERSAL SYMMETRY}

In Sec. III we see that the weak localization correction to $R_{p}(\omega)$ at the two loop level originates from the coupling between a diffuson and a cooperon. In the broken time-reversal systems, the cooperon modes are suppressed by the applied magnetic field. As a result, $Q$ takes the components $k=0,3$ only in the $d$ space [see Eq. (20)]. No longer exist the contraction rules Eqs. (33) and (34). Instead, we can find the contraction rules below:

$$
\begin{aligned}
4 \pi \nu \overbrace{P(1) M} P(2)= & \mathcal{D}^{0}(\overline{1}, \overline{2}) \Lambda_{\|}^{+} \operatorname{STr}\left[M \Lambda_{\|}^{-}\right]+\mathcal{D}^{0}(1,2) \Lambda_{\|}^{-} \mathrm{S} \operatorname{Tr}\left[M \Lambda_{\|}^{+}\right]+ \\
& \mathcal{D}^{0}(\overline{1}, \overline{2}) \Lambda_{\|}^{+} \tau_{3} \mathrm{~S} \operatorname{Tr}\left(M \Lambda_{\|}^{-} \tau_{3}\right)+\mathcal{D}^{0}(1,2) \Lambda_{\|}^{-} \tau_{3} \operatorname{STr}\left(M \Lambda_{\|}^{+} \tau_{3}\right),
\end{aligned}
$$

and

$$
2 \pi \nu \mathrm{S} \operatorname{Tr}[M \overbrace{P(1)] \mathrm{S} \operatorname{Tr}[N} P(2)]=\operatorname{S} \operatorname{Tr}\left[\left(\mathcal{D}^{0}(1,2) M-\mathcal{D}^{0}(\overline{1}, 2) \bar{M}\right) \Lambda_{\|}^{-} N \Lambda_{\|}^{+}+\left(\mathcal{D}^{0}(\overline{1}, \overline{2}) M-\mathcal{D}^{0}(1, \overline{2}) \bar{M}\right) \Lambda_{\|}^{+} N \Lambda_{\|}^{-}\right]
$$


by direct calculations. Then applying the Wick's theorem and the contraction rules to Eq. (32), we find that it vanishes as expected.

In the disordered case, it was first found that the leading weak localization corrections to $R_{p}(\omega)$ in the broken time-reversal system are at the three loop level by using the replica technique ${ }^{11}$. To our best knowledge, the weak localization of $R_{p}(\omega)$ in the quantum chaotic systems with the broken time-reversal symmetry has not yet been studied. In this section, we prove that for the latter case, $R_{p}(\omega)$ can be expressed as the second derivative of the free energy at the three loop level. Moreover, we find that the $(\Delta / \omega)^{4}$ term is not exactly zero as expected by the universal Wigner-Dyson statistics. Instead, it is an Ehrenfest oscillation, which shows a crossover to its universal limit at $\omega \lesssim t_{E}^{-1}$.

\section{A. Weak localization corrections to the perturbative $R(\omega)$}

From Eqs. (10), (21) and (25), we can find that $R_{p}(\omega)$ in the order of $(\Delta / \omega)^{4}$ is

$$
\Delta R_{p}^{\mathrm{u}}(\omega)=\Delta R_{p, 3 b}^{\mathrm{u}}(\omega)+\Delta R_{p, 3 d}^{\mathrm{u}}(\omega)
$$

Here

$$
\begin{aligned}
\Delta R_{p, 3 b}^{\mathrm{u}}(\omega)= & \frac{1}{16} \operatorname{Re} \int D P e^{-S^{0}} \int d 1 \mathrm{~S} \operatorname{Tr}\left[k P^{2}(1)\right] \int d 2 \mathrm{STr}\left[k P^{6}(2)\right]+ \\
& \frac{1}{16} \operatorname{Re} \int D P e^{-S^{0}} \int d 1 \mathrm{~S} \operatorname{Tr}\left[k P^{2}(1)\right] \int d 2 \mathrm{~S} \operatorname{Tr}\left[k P^{2}(2)\right] \cdot\left(\frac{1}{2} i \pi \nu \omega\right) \int d 3 \mathrm{STr}\left[P^{6}(3)\right]
\end{aligned}
$$

where we keep the term with $j=2$ in the effective interaction $S^{\text {int }}$.

$$
\begin{aligned}
\Delta R_{p, 3 d}^{\mathrm{u}}(\omega)= & \frac{1}{16} \operatorname{Re} \int D P e^{-S^{0}} \int d 1 \mathrm{STr}\left[k P^{4}(1)\right] \int d 2 \mathrm{~S} \operatorname{Tr}\left[k P^{4}(2)\right]+ \\
& \frac{1}{16} \operatorname{Re} \int D P e^{-S^{0}} \int d 1 \mathrm{~S} \operatorname{Tr}\left[k P^{2}(1)\right] \int d 2 \mathrm{~S} \operatorname{Tr}\left[k P^{4}(2)\right] \cdot\left(\frac{1}{2} i \pi \nu \omega\right) \int d 3 \mathrm{STr}\left[P^{4}(3)\right]+ \\
& \frac{1}{32} \operatorname{Re} \int D P e^{-S^{0}} \int d 1 \mathrm{~S} \operatorname{Tr}\left[k P^{2}(1)\right] \int d 2 \mathrm{~S} \operatorname{Tr}\left[k P^{2}(2)\right] \cdot\left(\frac{1}{2} i \pi \nu \omega\right)^{2} \int d 3 \mathrm{STr}\left[P^{4}(3)\right] \int d 4 \mathrm{STr}\left[P^{4}(4)\right] .
\end{aligned}
$$

In Eq. (50), we consider up to the second order effective interaction kept with the term $j=1$.

First, we consider Eq. (49). The first term in Eq. (49) is found to be

$$
\begin{aligned}
\Delta R_{p, b 1}^{\mathrm{u}}(\omega) & =-\frac{3}{4}\left(\frac{\Delta}{\pi}\right)^{4} \int d 1 d 2\left\langle\mathcal{D}^{0}(1,2) \mathcal{D}^{0}(2,1) \mathcal{D}^{0}(2,2) \mathcal{D}^{0}(2,2)\right\rangle \\
& =\frac{3}{4}\left(\frac{\Delta}{\pi}\right)^{4} \int d 1\left\langle\left[\frac{\partial}{\partial(-i \omega)} \mathcal{D}^{0}(2,2)\right] \mathcal{D}^{0}(2,2) \mathcal{D}^{0}(2,2)\right\rangle
\end{aligned}
$$

by using Eqs. (46) and (47). This gives the contraction shown in (b1), Fig. 2. The other term in Eq. (49) gives

$$
\begin{aligned}
\Delta R_{p, b 2}^{\mathrm{u}}(\omega) & =\frac{3}{4}\left(\frac{\Delta}{\pi}\right)^{4}(-i \omega) \int d 1 d 2 d 3\left\langle\mathcal{D}^{0}(1,3) \mathcal{D}^{0}(3,1) \mathcal{D}^{0}(2,3) \mathcal{D}^{0}(3,2) \mathcal{D}^{0}(3,3)\right\rangle \\
& =\frac{3}{4}\left(\frac{\Delta}{\pi}\right)^{4}(-i \omega) \int d 3\left\langle\left[\frac{\partial}{\partial(-i \omega)} \mathcal{D}^{0}(3,3)\right]\left[\frac{\partial}{\partial(-i \omega)} \mathcal{D}^{0}(3,3)\right] \mathcal{D}^{0}(3,3)\right\rangle
\end{aligned}
$$

and

$$
\begin{aligned}
\Delta R_{p, b 3}^{\mathrm{u}}(\omega) & =\frac{3}{4}\left(\frac{\Delta}{\pi}\right)^{4}(-i \omega) \int d 1 d 2 d 3\left\langle\mathcal{D}^{0}(3,1) \mathcal{D}^{0}(1,2) \mathcal{D}^{0}(2,3) \mathcal{D}^{0}(3,3) \mathcal{D}^{0}(3,3)\right\rangle \\
& =\frac{3}{8}\left(\frac{\Delta}{\pi}\right)^{4}(-i \omega) \int d 3\left\langle\left[\frac{\partial^{2}}{\partial(-i \omega)^{2}} \mathcal{D}^{0}(3,3)\right] \mathcal{D}^{0}(3,3) \mathcal{D}^{0}(3,3)\right\rangle,
\end{aligned}
$$

which corresponds to two ways of contraction shown in (b2) and (b3) Fig. 2. Adding them together, we find the free energy $F_{3 b}(\omega)$ to be [cf. Eq. (45)] 


$$
F_{3 b}(\omega)=\frac{\Delta^{2}}{8 \pi^{2}}(-i \omega) \int d 1\left\langle\mathcal{D}^{0}(1,1) \mathcal{D}^{0}(1,1) \mathcal{D}^{0}(1,1)\right\rangle .
$$

Now we turn to consider Eq. (50). The first term in Eq. (50) is reduced into

$$
\Delta R_{p, d 1}^{\mathrm{u}}(\omega)=-\frac{1}{4}\left(\frac{\Delta}{\pi}\right)^{4} \int d 1 d 2\left\langle\mathcal{D}^{0}(1,2) \mathcal{D}^{0}(1,2) \mathcal{D}^{0}(2,1) \mathcal{D}^{0}(2,1)\right\rangle
$$

by using Eqs. (46) and (47), which gives the contraction shown in (d1), Fig. 3. The second term in Eq. (50) is

$$
\begin{aligned}
\Delta R_{p, d 2}^{\mathrm{u}}(\omega) & =2\left(\frac{\Delta}{\pi}\right)^{4}(-i \omega) \int d 1 d 2 d 3\left\langle\mathcal{D}^{0}(2,3) \mathcal{D}^{0}(2,3) \mathcal{D}^{0}(3,2) \mathcal{D}^{0}(3,1) \mathcal{D}^{0}(1,2)\right\rangle \\
& =-2\left(\frac{\Delta}{\pi}\right)^{4}(-i \omega) \int d 2 d 3\left\langle\mathcal{D}^{0}(2,3) \mathcal{D}^{0}(2,3) \mathcal{D}^{0}(3,2)\left[\frac{\partial}{\partial(-i \omega)} \mathcal{D}^{0}(3,2)\right]\right\rangle
\end{aligned}
$$

which gives the contraction shown in (d2), Fig. 3. The diagram (d3) and (d4) in Fig. 3 come from the third term in Eq. (50):

$$
\begin{aligned}
\Delta R_{p, d 3}^{\mathrm{u}}(\omega)= & -\left(\frac{\Delta}{\pi}\right)^{4}(-i \omega)^{2} \int d 1 d 2 d 3 d 4\left\langle\mathcal{D}^{0}(3,4) \mathcal{D}^{0}(3,4) \mathcal{D}^{0}(4,3) \mathcal{D}^{0}(4,2) \mathcal{D}^{0}(2,1) \mathcal{D}^{0}(1,3)\right\rangle \\
= & -\frac{1}{2}\left(\frac{\Delta}{\pi}\right)^{4}(-i \omega)^{2} \int d 3 d 4\left\langle\mathcal{D}^{0}(3,4) \mathcal{D}^{0}(3,4) \mathcal{D}^{0}(4,3)\left[\frac{\partial^{2}}{\partial(-i \omega)^{2}} \mathcal{D}^{0}(4,3)\right]\right\rangle, \\
\Delta R_{p, d 4}^{\mathrm{u}}(\omega)= & -\left(\frac{\Delta}{\pi}\right)^{4}(-i \omega)^{2} \int d 1 d 2 d 3 d 4\left\langle\mathcal{D}^{0}(3,2) \mathcal{D}^{0}(2,4) \mathcal{D}^{0}(3,4) \mathcal{D}^{0}(4,3) \mathcal{D}^{0}(4,1) \mathcal{D}^{0}(1,3)\right\rangle- \\
& \frac{1}{2}\left(\frac{\Delta}{\pi}\right)^{4}(-i \omega)^{2} \int d 1 d 2 d 3 d 4\left\langle\mathcal{D}^{0}(3,1) \mathcal{D}^{0}(1,4) \mathcal{D}^{0}(4,3) \mathcal{D}^{0}(3,2) \mathcal{D}^{0}(2,4) \mathcal{D}^{0}(4,3)\right\rangle \\
= & -\left(\frac{\Delta}{\pi}\right)^{4}(-i \omega)^{2} \int d 3 d 4\left\langle\left[\frac{\partial}{\partial(-i \omega)} \mathcal{D}^{0}(3,4)\right] \mathcal{D}^{0}(3,4) \mathcal{D}^{0}(4,3)\left[\frac{\partial}{\partial(-i \omega)} \mathcal{D}^{0}(4,3)\right]\right\rangle- \\
& \frac{1}{2}\left(\frac{\Delta}{\pi}\right)^{4}(-i \omega)^{2} \int d 3 d 4\left\langle\left[\frac{\partial}{\partial(-i \omega)} \mathcal{D}^{0}(3,4)\right]\left[\frac{\partial}{\partial(-i \omega)} \mathcal{D}^{0}(3,4)\right] \mathcal{D}^{0}(4,3) \mathcal{D}^{0}(4,3)\right\rangle .
\end{aligned}
$$

Collecting Eqs. (55)-(58) together, we find the free energy $F_{3 d}(\omega)$ to be [cf. Eq. (45)]

$$
F_{3 d}(\omega)=-\frac{\Delta^{2}}{8 \pi^{2}}(-i \omega)^{2} \int d 1 d 2\left\langle\mathcal{D}^{0}(1,2) \mathcal{D}^{0}(1,2) \mathcal{D}^{0}(2,1) \mathcal{D}^{0}(2,1)\right\rangle .
$$

Hence we prove Eq. (45) at the three loop level. We see that Eqs. (49) and (50) give the weak localization correction to $R_{p}(\omega)$, up to the order of $(\Delta / \omega)^{4}$ as

$$
\Delta R_{p}^{\mathrm{u}}(\omega)=\left(\frac{\Delta}{\pi}\right)^{2} \operatorname{Re} \frac{\partial^{2}}{\partial(-i \omega)^{2}}\left(F_{3 b}(\omega)+F_{3 d}(\omega)\right)
$$

\section{B. The Couplings of Diffusons}

In this subsection we will calculate the product of diffusons appearing in Eqs. (54) and (59).

We emphasize that it is difficult to use the standard diagram technique ${ }^{45}$ to calculate these quantities. Instead, it is more convenient to use the semiclassical method.
In this section, we will follow the techniques of Ref. 22 . This immediately leads to the Ehrenfest oscillations in the perturbative $R(\omega)$. It is important that the average $\langle\cdots\rangle$ is performed over the energy for fixed potentials, not impurity configurations.

\section{The couplings of two diffusons}

To calculate Eq. (59), we notice that there is the following relation:

$$
\begin{aligned}
& \left\langle\mathcal{D}^{0}(1,2) \mathcal{D}^{0}(1,2) \mathcal{D}^{0}(2,1) \mathcal{D}^{0}(2,1)\right\rangle \\
= & \left\langle\mathcal{D}^{0}(1,2) \mathcal{D}^{0}(1,2)\right\rangle\left\langle\mathcal{D}^{0}(2,1) \mathcal{D}^{0}(2,1)\right\rangle
\end{aligned}
$$

for similar reasons as discussions for Eq. (42). Actually $\mathcal{D}^{0}(1,2)$ stands for the probability of a trajectory initi- 
ating from 1 and ending at 2 in the phase space, while $\mathcal{D}^{0}(2,1)$ stands for the probability of a trajectory initiating from 2 and ending at 1 . Thus such two trajectories are distant parts and decoupled. But $\left\langle\mathcal{D}^{0}(1,2) \mathcal{D}^{0}(1,2)\right\rangle$ (b1)

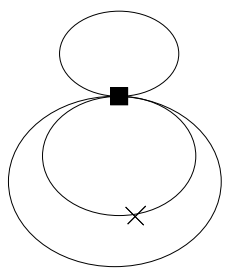

(b3)

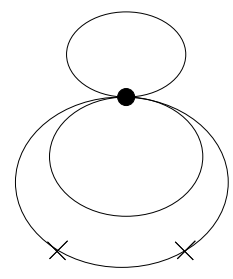

(b2)

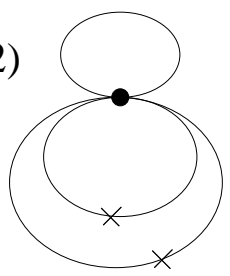

- $=-\mathrm{i} \omega$

- $=\times=1$

$=\mathrm{D}^{0}(1,2)$
FIG. 2. The diagrams with 6-point vertex contribute to $R_{p}(\omega)$ in the order of $(\Delta / \omega)^{4}$ in systems with broken time-reversal symmetry. The cross and the square, $\sim \operatorname{Str}\left[k P^{2}\right]$ and $\sim \operatorname{Str}\left[k P^{6}\right]$, respectively, come from the expansion in the prefactor, see Eq. (21). The dot, $\sim i \omega \operatorname{Str}\left[P^{6}\right]$, comes from the effective interaction, see Eq. (25). In the figure, we put $\Delta=1$.

can not be factorized at the region near 1 or 2 , where two trajectories become very close to each other.

We will study the quantity $\left\langle\mathcal{D}^{0}\left(1_{1}, 2\right) \mathcal{D}^{0}\left(1_{2}, 2\right)\right\rangle$ instead of $\left\langle\mathcal{D}^{0}(1,2) \mathcal{D}^{0}(1,2)\right\rangle$ and put $1_{1}=1_{2}$ in the final answer. Here we use the subscripts 1 and 2 to denote the small deviations in the momentum direction and position. From Eq. (35), we find the motion equation to be

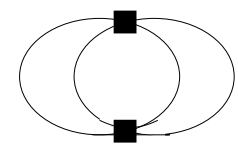

(d1)
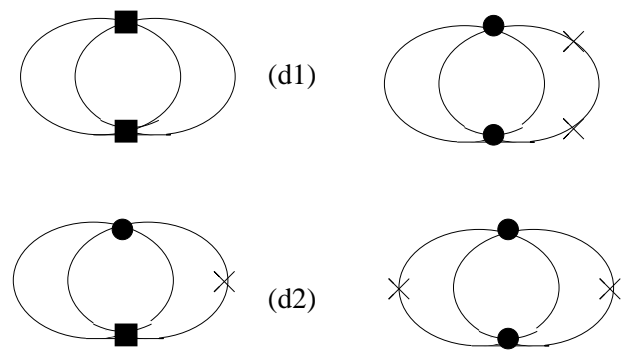

(d2)

$=\mathrm{i} \omega$

$$
=\mathrm{D}^{0}(1,2)
$$

FIG. 3. The diagrams with 4-point vertex contribute to $R_{p}(\omega)$ in the order of $(\Delta / \omega)^{4}$ in systems with broken time-reversal symmetry. The cross and the square, $\sim \operatorname{Str}\left[k P^{2}\right]$ and $\sim \operatorname{Str}\left[k P^{4}\right]$, respectively, come from the expansion in the prefactor, see Eq. (21). The dot, $\sim i \omega \operatorname{Str}\left[P^{4}\right]$, comes from the effective interaction, see Eq. (25). In the figure, we put $\Delta=1$.

$$
\left(-2 i \omega+\hat{\mathcal{L}}_{1_{1}}+\hat{\mathcal{L}}_{1_{2}}-\frac{1}{\tau_{q}} \frac{\partial^{2}}{\partial \phi_{1_{1}}^{2}}-\frac{1}{\tau_{q}} \frac{\partial^{2}}{\partial \phi_{1_{2}}^{2}}\right) \mathcal{D}^{0}\left(1_{1}, 2\right) \mathcal{D}^{0}\left(1_{2}, 2\right)=2 \pi\left[\delta_{1_{1}, 2} \mathcal{D}^{0}\left(1_{2}, 2\right)+\delta_{1_{2}, 2} \mathcal{D}^{0}\left(1_{1}, 2\right)\right]
$$

We will use $\left(\mathbf{n}_{1}, \mathbf{R}_{1}\right)$ to denote the motion of the center of mass while $\left(\phi_{1}, \rho_{1}\right)$ to denote the relative motion ${ }^{22}$. The operator $\left(\hat{\mathcal{L}}_{1_{1}}+\hat{\mathcal{L}}_{1_{2}}\right)$ is written as

$$
\begin{array}{r}
\hat{\mathcal{L}}_{1}+\hat{\mathcal{L}}_{2}=\hat{\mathcal{L}}_{c, 1}+\hat{\mathcal{L}}_{r, 1} \\
\hat{\mathcal{L}}_{c, 1}=v_{F} \mathbf{n}_{1} \cdot \frac{\partial}{\partial \mathbf{R}_{1}}-\nabla U\left(\mathbf{R}_{1}\right) \cdot \frac{\partial}{\partial \mathbf{p}_{1}}, \\
\hat{\mathcal{L}}_{r, 1}=-v_{F} \phi_{1} \frac{\partial}{\partial \rho_{1}}+\frac{\partial^{2} U}{p_{F} \partial R_{\perp, 1}^{2}} \rho_{1} \frac{\partial}{\partial \phi_{1}},
\end{array}
$$

where $R_{\perp}$ denote the coordinate perpendicular to the momentum direction. For the convenience below, we further introduce the change of variables:

$$
\begin{aligned}
& z_{1}=\ln \left[\phi_{1}^{2}+\left(\frac{\rho_{1}}{L}\right)^{2}\right]^{1 / 2}, \quad \alpha_{1}=\arctan \frac{\phi_{1} L}{\rho_{1}} . \\
& {\left[2 i \omega+\lambda \frac{\partial}{\partial z_{1}}+\frac{\lambda_{2}}{2} \frac{\partial^{2}}{\partial z_{1}^{2}}+\frac{e^{-2 z_{1}}}{2 \tau_{q}} \frac{\partial}{\partial z_{1}}\left(\frac{1-\gamma}{2} \frac{\partial}{\partial z_{1}}+\gamma\right)\right] M\left(z_{1} ; 2,2\right)=0 .}
\end{aligned}
$$

At $\omega \ll \lambda$, the deterministic motion of the center of mass becomes random. Thus, the quantity $\mathcal{D}^{0}\left(1_{1}, 2\right) \mathcal{D}^{0}\left(1_{2}, 2\right)$ is self-averaging over $\mathbf{R}_{1}, \mathbf{n}_{1}$. In this way, we can introduce the following quantity:

$$
\begin{aligned}
M\left(\phi_{1}, \rho_{1} ; 2,2\right) & \equiv\left\langle\mathcal{D}^{0}\left(1_{1}, 2\right) \mathcal{D}^{0}\left(1_{2}, 2\right)\right\rangle \\
& =\int \frac{d \mathbf{R}_{1} d \mathbf{n}_{1}}{2 \pi A} \mathcal{D}^{0}\left(1_{1}, 2\right) \mathcal{D}^{0}\left(1_{2}, 2\right) .
\end{aligned}
$$

By definition we imply that $\left|z_{1}\right| \simeq\left|z_{2}\right| \gg 1$. In this way, we can see that the particular solution due to the r.h.s. of Eq. (62) is of the order of $1 / \lambda \omega$ and depends on $z_{1}-z_{2} \simeq 0$. Therefore, we can ignore the r.h.s. of Eq. (62) since the particular solution is much smaller than $1 / \omega^{2}$ in the universal region: $\omega \ll \lambda$. Then averaging Eq. (63) 
Eq. (66) exists only at the Lyapunov region, i.e., $\rho \ll$ $L, \phi \ll 1\left(z_{1} \ll-1\right)$. At $z_{1} \sim 0$, Eq. (66) is not applicable and the solution of $M$ does not depend on $z_{1}$ any more. Since we are interested in the region $\omega \sim t_{E}^{-1}$, the coordinates 1 and 2 are independent from each other. Hence $z_{1} \simeq z_{2}$, by definition, implies that the deterministic relative motion (Lyapunov asymptotic instability) of $z_{1}$ must reach $z_{1}=0$ in the course of time. In this way, Eq. (66) must be supplemented by the boundary condition $M\left(\omega, z_{1}=0\right)=M^{\prime} . M^{\prime}$ will be calculated below.

The solution of Eq. (66) is

$$
M=w_{2}\left(\omega, z_{1}\right) M^{\prime},
$$

where $w_{2}\left(\omega, z_{1}\right)$ is found to $\mathrm{be}^{22}$

$$
w_{2}\left(\omega, z_{1}\right)=\exp \left[\left(\frac{i \omega}{\lambda}-\frac{\omega^{2} \lambda_{2}}{\lambda^{3}}\right) \ln \frac{\lambda \tau_{q}}{\lambda \tau_{q} e^{2 z_{1}}+\gamma / 2}\right] \text {. }
$$

up to the logarithmic accuracy. $\gamma \lesssim 1$ is a numerical factor. Any other form of the regularizer with the form of the second order elliptic operator changes the value of $\gamma$ only. $t_{E}$ is the Ehrenfest time:

$$
t_{E}=\frac{1}{\lambda}\left|\ln \lambda \tau_{q}\right|
$$

In the limit $z_{1} \rightarrow-\infty, M$ becomes

$$
M=\Gamma_{2}(\omega) M^{\prime} .
$$

At the classical limit $\tau_{q} \rightarrow \infty, w_{2} \rightarrow 0$ as $z_{1} \rightarrow-\infty$. But this is not the case for finite $\tau_{q}$. As we see from Eq. (68), the classical solution $w_{2}\left(\omega, z_{1}\right)\left(\tau_{q}=\infty\right)$ thereby acquires a lower cutoff at $z_{1}=-\frac{1}{2} \ln \lambda \tau_{q}$. That is, we may regard $w_{2}(\omega,-\infty)$ as $w_{2}\left(\omega,-\frac{1}{2} \ln \lambda \tau_{q}\right)$.

To find $M^{\prime}$, we repeat the procedure of deriving Eq. (62) and study a more general quantity: $U\left(\omega ; \overline{2}_{1}, \overline{2}_{2}\right), M^{\prime}=U(\omega ; \overline{2}, \overline{2})$. Similarly to Eq. (66), $U\left(z_{\overline{2}} ; \omega\right)$ is the solution of the equation:

$$
\left[2 i \omega+\lambda \frac{\partial}{\partial z_{\overline{2}}}+\frac{\lambda_{2}}{2} \frac{\partial^{2}}{\partial z_{\overline{2}}^{2}}+\frac{e^{-2 z_{\overline{2}}}}{2 \tau_{q}} \frac{\partial}{\partial z_{\overline{2}}}\left(\frac{1-\gamma}{2} \frac{\partial}{\partial z_{\overline{2}}}+\gamma\right)\right] U\left(z_{\overline{2}} ; \omega\right)=0
$$

where $z_{\overline{2}}=z_{2}$ characterizes the separation of two nearby trajectories centered at $\overline{2}$. We drop out the r.h.s. for the same reasons as deriving Eq. (66). To find the solution of Eq. (71). We repeat the procedure of Eqs. (67) to (70), except that the boundary condition is replaced by

$$
U\left(z_{\overline{2}}=0, \omega\right)=\frac{1}{(-i \omega)^{2}}
$$

Eq. (72) means that if the deviations $\rho_{1} \sim \rho_{2} \sim L\left(z_{1}=\right.$ $\left.z_{2}=0\right)$, then the two trajectories, connecting $1_{1}$ and $2_{1}$, $1_{2}$ and $2_{2}$, respectively, are independent from each other. Thus we have $\left\langle\mathcal{D}^{0} \mathcal{D}^{0}\right\rangle=\left\langle\mathcal{D}^{0}\right\rangle^{2}$. Similarly, we have

$$
M^{\prime}=U\left(z_{2}=-\infty\right)=\Gamma_{2}(\omega) \frac{1}{(-i \omega)^{2}}
$$

Substituting Eq. (73) into Eq. (70), we find that

$$
\left\langle\mathcal{D}^{0}(1,2) \mathcal{D}^{0}(1,2)\right\rangle \equiv M=\frac{\Gamma_{2}^{2}(\omega)}{(-i \omega)^{2}}
$$

At $\omega \ll \lambda$,

$$
\begin{gathered}
\left(-3 i \omega+\hat{\mathcal{L}}_{1_{1}}+\hat{\mathcal{L}}_{1_{2}}+\hat{\mathcal{L}}_{1_{3}}-\frac{1}{\tau_{q}} \frac{\partial^{2}}{\partial \phi_{1_{1}}^{2}}-\frac{1}{\tau_{q}} \frac{\partial^{2}}{\partial \phi_{1_{2}}^{2}}-\frac{1}{\tau_{q}} \frac{\partial^{2}}{\partial \phi_{1_{3}}^{2}}\right) \mathcal{D}^{0}\left(1_{1}, 2\right) \mathcal{D}^{0}\left(1_{2}, 2\right) \mathcal{D}^{0}\left(1_{3}, 2\right) \\
=2 \pi\left[\delta_{1_{1}, 2} \mathcal{D}^{0}\left(1_{2}, 2\right) \mathcal{D}^{0}\left(1_{3}, 2\right)+\delta_{1_{2}, 2} \mathcal{D}^{0}\left(1_{1}, 2\right) \mathcal{D}^{0}\left(1_{3}, 2\right)+\delta_{1_{3}, 2} \mathcal{D}^{0}\left(1_{1}, 2\right) \mathcal{D}^{0}\left(1_{2}, 2\right)\right]
\end{gathered}
$$

Then we can separate the motion into the part of the center of mass and the relative part by introducing the change of variables:

$$
\left\langle\mathcal{D}^{0}(1,1) \mathcal{D}^{0}(1,1)\right\rangle=\left\langle\mathcal{D}^{0}(1,2) \mathcal{D}^{0}(1,2)\right\rangle=\frac{\Gamma_{2}^{2}(\omega)}{(-i \omega)^{2}}
$$

We would like to mention that in the case of longranged disorders with $\tau_{q}=\infty$ ), an equation similar to Eq. (66) was found by using the path integral technique ${ }^{46}$. In fact, in this case, $\lambda$ and $\lambda_{2}$ were calculated [see Appendix of Ref. 22]. It is found that $\lambda_{2} \simeq 4 \lambda$.

\section{The couplings of three diffusons}

Now we turn to calculate Eq. (54). First, we note that although the coordinates in $\mathcal{D}^{0}(1,1)$ coincide with each other, they are not related to each other at the region $\omega \ll \lambda$, because the trajectory travels for a long time before returning to the same position in the phase space. Thus, to calculate $\left\langle\mathcal{D}^{0}(1,1) \mathcal{D}^{0}(1,1) \mathcal{D}^{0}(1,1)\right\rangle$ in Eq. (54), we will calculate a more general quantity, say $\left\langle\mathcal{D}^{0}(1,2) \mathcal{D}^{0}(1,2) \mathcal{D}^{0}(1,2)\right\rangle$.

We proceed along the line of calculating two coupled diffusons. Similarly to Eq. (62), we have the following equation: 


$$
\begin{aligned}
& \mathbf{R}_{1}=\frac{\mathbf{R}_{1_{1}}+\mathbf{R}_{1_{2}}+\mathbf{R}_{1_{3}}}{3}, \quad \phi_{1}=\frac{\phi_{1_{1}}+\phi_{1_{2}}+\phi_{1_{3}}}{3}, \\
& \mathbf{r}_{1_{1}}=\mathbf{R}_{1_{1}}-\mathbf{R}_{1}, \quad \theta_{1}=\phi_{1_{1}}-\phi_{1}, \\
& \mathbf{r}_{1_{2}}=\mathbf{R}_{1_{2}}-\mathbf{R}_{1}, \quad \theta_{2}=\phi_{1_{2}}-\phi_{1} .
\end{aligned}
$$

Here $\left(\phi_{1}, \mathbf{R}_{1}\right)$ denotes the coordinates of the center of mass. The relative motion is described by the set of variables: $\left(\mathbf{r}_{1_{1}}, \mathbf{r}_{1_{2}} ; \theta_{1}, \theta_{2}\right)$. The transverse components of $\mathbf{r}_{1_{1}}$ and $\mathbf{r}_{1_{2}}$ are denoted as $\rho_{1}$ and $\rho_{2}$. Under such a change of variables, we rewrite Eq. (76) as

$$
\begin{aligned}
& {\left[-3 i \omega+\hat{\mathcal{L}}_{\mathbf{R}_{1}}+\hat{\mathcal{L}}_{\mathbf{r}_{1_{1}}}+\hat{\mathcal{L}}_{\mathbf{r}_{1_{2}}}-\frac{1}{3 \tau_{q}} \frac{\partial^{2}}{\partial \phi_{1}^{2}}-\frac{2}{3} \frac{1}{\tau_{q}}\left(\frac{\partial^{2}}{\partial \theta_{1}^{2}}+\frac{\partial^{2}}{\partial \theta_{2}^{2}}-\frac{\partial^{2}}{\partial \theta_{1} \partial \theta_{2}}\right)\right] \mathcal{D}^{0}\left(1_{1}, 2\right) \mathcal{D}^{0}\left(1_{2}, 2\right) \mathcal{D}^{0}\left(1_{3}, 2\right)=0} \\
& \hat{\mathcal{L}}_{\mathbf{R}_{1}}=v_{F} \mathbf{n}_{1} \cdot \frac{\partial}{\partial \mathbf{R}_{1}}-\nabla U\left(\mathbf{R}_{1}\right) \cdot \frac{\partial}{\partial \mathbf{p}_{1}} \\
& \hat{\mathcal{L}}_{\mathbf{r}_{1_{1}}}=-v_{F} \theta_{1} \frac{\partial}{\partial \rho_{1}}+\frac{\partial^{2} U}{p_{F} \partial R_{\perp, 1}^{2}} \rho_{1} \frac{\partial}{\partial \theta_{1}} \\
& \hat{\mathcal{L}}_{\mathbf{r}_{1_{2}}}=-v_{F} \theta_{2} \frac{\partial}{\partial \rho_{2}}+\frac{\partial^{2} U}{p_{F} \partial R_{\perp, 1}^{2}} \rho_{2} \frac{\partial}{\partial \theta_{2}}
\end{aligned}
$$

Here we expand $\theta, \rho$ up to the first order. We again drop out the r.h.s. of Eq. (76). In Eq. (78), the form of the regularizer has changed compared to Eq. (62). However, as we discussed in Sec. II A, such a change accounts only for the value of $\tau_{q}$ under logarithm. Consequently, up to the logarithmic accuracy, $t_{E}$ remains unchanged. In view of this, we ignore the regularizer in the discussions below. To solve Eq. (78), we will proceed along the line of calculating two coupled diffusons. Here we will show the main result and give the details in Appendix A. Eq. (78) can be reduced as

$$
\begin{aligned}
& \left(3 i \omega+\lambda \frac{\partial}{\partial x}+\frac{\lambda_{2}}{2} \frac{\partial^{2}}{\partial x^{2}}\right) N(x ; 2,2)=0, \\
& x=\frac{1}{2}\left(\ln \sqrt{\theta_{1}^{2}+\left(\frac{\rho_{1}}{L}\right)^{2}}+\ln \sqrt{\theta_{2}^{2}+\left(\frac{\rho_{2}}{L}\right)^{2}}\right),
\end{aligned}
$$

where $N(x ; 2,2)$ is the self-averaging of $\mathcal{D}^{0}\left(1_{1}, 2\right) \mathcal{D}^{0}\left(1_{2}, 2\right) \mathcal{D}^{0}\left(1_{3}, 2\right)$ over the center of mass $\mathbf{R}_{1}, \mathbf{n}_{1}$ :

$$
\begin{aligned}
& N(x ; 2,2)=N(\theta, \rho ; 2,2) \\
\equiv & \left\langle\mathcal{D}^{0}\left(1_{1}, 2\right) \mathcal{D}^{0}\left(1_{2}, 2\right) \mathcal{D}^{0}\left(1_{3}, 2\right)\right\rangle \\
= & \int \frac{d \mathbf{R}_{1} d \mathbf{n}_{1}}{2 \pi A} \mathcal{D}^{0}\left(1_{1}, 2\right) \mathcal{D}^{0}\left(1_{2}, 2\right) \mathcal{D}^{0}\left(1_{3}, 2\right) .
\end{aligned}
$$

The similar equation was proposed in Ref. 26. It is important that here $\lambda$ and $\lambda_{2}$ stay exactly the same as those appearing in $\Gamma_{2}(\omega)$ (see Appendix A). Compared to Eq. (66), Eq. (79) differs from it in the frequency $\left(\omega \rightarrow \frac{3}{2} \omega\right)$ and the variable $(z \rightarrow x)$. Therefore, in the limit $\tau_{q} \rightarrow \infty$, the homogeneous solution of Eq. (79), say $w_{3}(x ; \omega)$ is

$$
w_{3}(x ; \omega)=w_{2}\left(x ; \frac{3}{2} \omega\right)
$$

supplemented by the boundary condition, which is $w_{3}(x=0 ; \omega)=1$. Up to the logarithmic accuracy, we obtain:

$$
\begin{aligned}
\Gamma_{3}(\omega) & \equiv \lim _{x \rightarrow-\infty} w_{3}(x ; \omega)=w_{3}\left(-\lambda^{-1} \ln \lambda \tau_{q} ; \omega\right) \\
& =\exp \left(\frac{3}{2} i \omega t_{E}-\frac{9}{4} \frac{\omega^{2} \lambda_{2} t_{E}}{\lambda^{2}}\right) .
\end{aligned}
$$

Then we follow the procedure of Eqs. (67)-(74) to get:

$$
\left\langle\mathcal{D}^{0}(1,2) \mathcal{D}^{0}(1,2) \mathcal{D}^{0}(1,2)\right\rangle=N(x \rightarrow-\infty, \omega)=\frac{\Gamma_{3}^{2}(\omega)}{(-i \omega)^{3}} .
$$

In fact, we have a general expression for $n$-coupled diffusons, say $\Gamma_{n}(\omega)$. One is able to prove that $\Gamma_{n}(\omega)$ is also oscillating: $\sim \exp \left(i n \omega t_{E} / 2\right)$ in the same spirit, which can be obtained in an alternative method, i.e., averaging the classical solution $\left(\tau_{q} \rightarrow \infty\right)$ over a minimal Gaussian wave packet ${ }^{26}$. It is in order to make the following observations: For $\Gamma_{n}(\omega)$, the oscillating part does not depend on the particular choice of the regularizer except that $t_{E}$ depends on $\tau_{q}$ parametrically.

\section{The Ehrenfest oscillations in $R_{p}^{\mathrm{u}}(\omega)$}

$F_{3 b}(\omega)$ and $F_{3 d}(\omega)$ are the ballistic counterparts of the zero mode approximation of the free energy in disordered systems. In the disordered limit or $\Delta \ll \omega \ll t_{E}^{-1}$, one can verify that $F_{3 b}(\omega)$ and $F_{3 d}(\omega)$ cancel each other and Eq. (60) gives vanishing result for $\Delta R_{p}^{\mathrm{u}}(\omega)$ by putting $\mathcal{D}^{0}(1,2)$ to be zero mode diffuson, i.e. $\mathcal{D}^{0}(1,2)=1 /-i \omega$. However, as we will see below, it is not so for chaotic quantum dots in the crossover region $t_{E}^{-1} \sim \omega \ll t_{\text {erg }}^{-1}$. Instead, Eqs. (54), (59) and (60) lead to the Ehrenfest oscillation with the period $\sim t_{E}^{-1}$. 
Substituting Eq. (83) into Eq. (54), we find that

$$
F_{3 b}(\omega)=\frac{\Delta^{2}}{8 \pi^{2}} \frac{\Gamma_{3}^{2}(\omega)}{(-i \omega)^{2}}
$$

Taking Eq. (61) into account, we find

$$
F_{3 d}(\omega)=-\frac{\Delta^{2}}{8 \pi^{2}} \frac{\Gamma_{2}^{4}(\omega)}{(-i \omega)^{2}} .
$$

Therefore, we obtain Eq. (7). We see that $R_{p}^{\mathrm{u}}(\omega)$ acquires an oscillation correction at $\omega t_{E} \sim 1$ with the pe$\operatorname{riod} \sim t_{E}^{-1}$.

We should emphasize that the appearance of the Ehrenfest oscillations, $\sim e^{i n \omega t_{E}}, n=1,2,3, \cdots$ do not depend on a particular form of the regularizer, although $t_{E}$ does. This can be understood in the following way. In the Lyapunov region, $n$ coupled diffusons (Hikami box with $2 n$-point vertex) contribute to $R(\omega)$ (in the disordered limit) an additional factor $\sim\left(G_{\epsilon+\frac{\omega}{2}}^{R} G_{\epsilon-\frac{\omega}{2}}^{A}\right)^{n}$. In the semiclassics, $G_{\epsilon}^{R} \sim e^{i \epsilon l / v_{F}}$ and $G_{\epsilon}^{A} \sim e^{-i \epsilon l / v_{F}}$ with $l$ being the length of the trajectory piece. Up to the logarithmic accuracy, the Lyapunov regions have the same length $v_{F} t_{E}$. In this way, $\left(G_{\epsilon+\frac{\omega}{2}}^{R} G_{\epsilon-\frac{\omega}{2}}^{A}\right)^{n} \sim e^{i n \omega t_{E}}$. In general, $R_{p}(\omega)$ can be expressed as

$$
\begin{aligned}
R_{p}(\omega) & =1+\sum_{n}\left(\frac{\Delta}{\omega}\right)^{n} C_{n}\left(\omega t_{E}\right) \\
& =1+\frac{\Delta^{2}}{\pi^{2}} \frac{\partial^{2}}{\partial \omega^{2}} F(\omega), \\
F(\omega) & =\sum_{L}\left(\frac{\Delta}{-i \omega}\right)^{L-1} \sum_{V, S} \delta_{L+V-S, 1} \tilde{C}_{S}\left(\omega t_{E}\right),
\end{aligned}
$$

where $L, V$ and $S$ characterize the topology of the diagram of the free energy, from which $R_{p}(\omega)$ is obtained. $L, S$ and $V$ are the number of loops, sides (diffusons) and vertices (Hikami boxes) respectively. The constraint comes from the well known Euler theorem in the topology ${ }^{47}$. For a topological diagram of the free energy with $S$ sides (diffusons), we have $\left(G_{\epsilon+\frac{\omega}{2}}^{R} G_{\epsilon-\frac{\omega}{2}}^{A}\right)^{S} \sim$ $e^{i S \omega t_{E}}$. In the systems with time-reversal symmetry, the leading corrections come from the two loop diagram, $L=2, S=2$ and $V=1$, hence the leading Ehrenfest oscillation in $R_{p}(\omega)$ is $\sim e^{i 2 \omega t_{E}}$. In systems with broken time-reversal symmetry, the leading corrections come from three loop diagrams. The diagram with the Hikami box of 6-point vertex $(L=3, S=3$ and $V=1)$ leads to the Ehrenfest oscillation $\sim e^{i 3 \omega t_{E}}$. The diagram with two Hikami boxes $(L=3, S=4$ and $V=2)$ leads to the Ehrenfest oscillation $\sim e^{i 4 \omega t_{E}}$.

\section{THE NONPERTURBATIVE PART OF $R(\omega)$ IN SYSTEMS WITH BROKEN TIME-REVERSAL SYMMETRY}

In Sec. III and IV, we study the perturbative expansion around the saddle point $\Lambda$. As a result, it gives the weak localization corrections to the leading perturbative term of the universal Wigner-Dyson statistics. However, it is known that such an expansion cannot reproduce the Wigner-Dyson oscillation ${ }^{33}$. Instead, Andreev and Altshuler found that such a nonperturbative term is controlled by other nontrivial saddle points ${ }^{33}$. In this section, we apply their method to study the leading order corrections to the universal Wigner-Dyson oscillation in the region $\Delta \ll \omega \sim t_{E}^{-1} \ll t_{\text {erg }}^{-1}$.

\section{A. The global transformation}

For GOE and GUE, the other saddle point is $-k \Lambda^{33}$. To establish a perturbation theory, a trick, so-called global transformation on $\mathbf{H}$ is introduced, which maps the saddle point $-k \Lambda$ to $\Lambda$. That is, we perform a global coordinate transformation:

$$
Q \rightarrow Q^{\prime}=U_{0}^{-1} Q U_{0}, \quad T \rightarrow T^{\prime}=T U_{0},
$$

where $U_{0} \in \mathbf{H}$ satisfies $-U_{0}^{-1} k \Lambda U_{0}=\Lambda$. It is important that the Jabobian is unity and $U_{0}$ does not depend on the coordinates. Under the transformation Eq. (87),

$$
Q^{\prime}=T^{\prime-1} \Lambda T^{\prime} .
$$

Thus, we can rewrite $R(\omega)$, Eq. (10) as

$$
R(\omega)=\frac{1}{64} \operatorname{Re} \int D Q^{\prime}\left(\int d x_{\|} \operatorname{STr}\left[\Lambda Q^{\prime}\left(x_{\|}\right)\right]\right)^{2} e^{-\tilde{S}_{e f f}},
$$

where $\tilde{S}_{\text {eff }}$ is the action:

$$
\tilde{S}_{e f f}=\frac{\pi \nu}{2} \int d x_{\|} \operatorname{STr}\left[-\frac{i \omega^{+}}{2} k \Lambda Q^{\prime}-T^{\prime-1} \Lambda \hat{\mathcal{L}} T^{\prime}+\frac{1}{\tau_{q}}\left(\frac{\partial Q^{\prime}}{\partial \phi}\right)^{2}\right] .
$$

Although the findings in Ref. 33 are asymptotic, it is important that the perturbation near the nontrivial saddle points take into account nonzero mode approximations, which, in principle, cannot be done by zero- dimensional supermatrix $\sigma$ model. Indeed, for $\mathrm{BNS} \sigma \mathrm{M}$, the Gaussian approximation is not applicable ${ }^{32}$ in the nonuniversal region: $\omega \gg t_{\text {erg }}^{-1}$. However, we believe that the perturbation near saddle points is applicable 
in the universal region $\omega \ll t_{e r g}^{-1}$, because the repetition problem ${ }^{17}$ is not essential in this case. In the ballistic case, the new region: $t_{E}^{-1} \sim \omega \ll t_{\text {erg }}^{-1}$, appears, within which the relaxation of the momentum is not complete. As a result, one can not put $Q$ to be a constant matrix. The parameterization, Eqs. (16)-(18) still holds for $T^{\prime}$. Furthermore, we can expand $B$ in terms of the Pauli's matrices:

$$
B=\sum_{k} B_{k} \otimes \tau_{d, k}, \quad \bar{B}=\sum_{k} \bar{B}_{k} \otimes \tau_{d, k} .
$$

The modes $k=0,3$ stand for the diffuson and the modes $k=1,2$ stand for the cooperon. They satisfy the same equation Eq. (35) in the absence of magnetic fields. In this way, we call both of them diffuson modes in this paper unless special explanation. The modes $k=1,2$ are suppressed in systems with broken time-reversal symmetry due to the destruction of the interference between a

trajectory and its time-reversed partner. In this paper, we will study only this case. Due to Eq. (14), the matrices $B_{k}$ and $\bar{B}_{k}$ satisfy:

$$
\bar{B}_{k}=k B_{k}^{\dagger}, \quad k=0,1,2,3 .
$$

$B_{k}$ may be parameterized as

$$
B_{k}=\left(\begin{array}{cc}
a_{k} & i \sigma_{k} \\
\eta_{k}^{*} & i b_{k}
\end{array}\right)
$$

where $a_{k}(1)$ and $b_{k}(1)$ are ordinary variables, while $\sigma_{k}(1)$ and $\eta_{k}(1)$ are Grassmann variables. Then we substitute Eqs. (91)-(93) into Eq. (89) and keep the quadratic terms in $a_{k}, b_{k}, \sigma_{k}$ and $\eta_{k}^{*}$ to rewrite Eq. (90) as

$$
\tilde{S}_{e f f}^{0}=-2 i \pi \omega+\tilde{S}^{0} .
$$

$\tilde{S}^{0}=2 \pi \int d x_{\|} \sum_{k=0,3}\left[a_{k}^{*}\left(x_{\|}\right)\left(i \omega^{+}+\hat{\mathcal{L}}_{R}\right) a_{k}\left(x_{\|}\right)+b_{k}^{*}\left(x_{\|}\right)\left(-i \omega^{+}+\hat{\mathcal{L}}_{R}\right) b_{k}\left(x_{\|}\right)+\sigma_{k}^{*}\left(x_{\|}\right) \hat{\mathcal{L}}_{R} \sigma_{k}\left(x_{\|}\right)+\eta_{k}\left(x_{\|}\right) \hat{\mathcal{L}}_{R} \eta_{k}^{*}\left(x_{\|}\right)\right]$

It is important to see that the new Gaussian action, $\tilde{S}^{0}$ does not include zero mode contribution of Grassmann fields. Thus, for a generic Gaussian integral with the action $\tilde{S}^{0}$ not to vanish, the prefactor must consist of all the independent zero mode Grassmann variables. In this way, by expanding $Q^{\prime}$ in the prefactor up to the second order, and taking into account the zero mode bosonic and Grassmann fields only, the universal Wigner-Dyson oscillation is reproduced in Refs. 30,33 .

\section{B. Quantum corrections}

The leading order corrections to the universal WignerDyson oscillation has the amplitude $\sim(\Delta / \omega)^{3}$ at $\omega \gg \Delta$. To see this, one need to expand $T^{\prime-1}$ and keep $Q$ up to $P^{4}$ and take into account the first order effective interaction similarly to Eq. (25). As a result, we obtain:

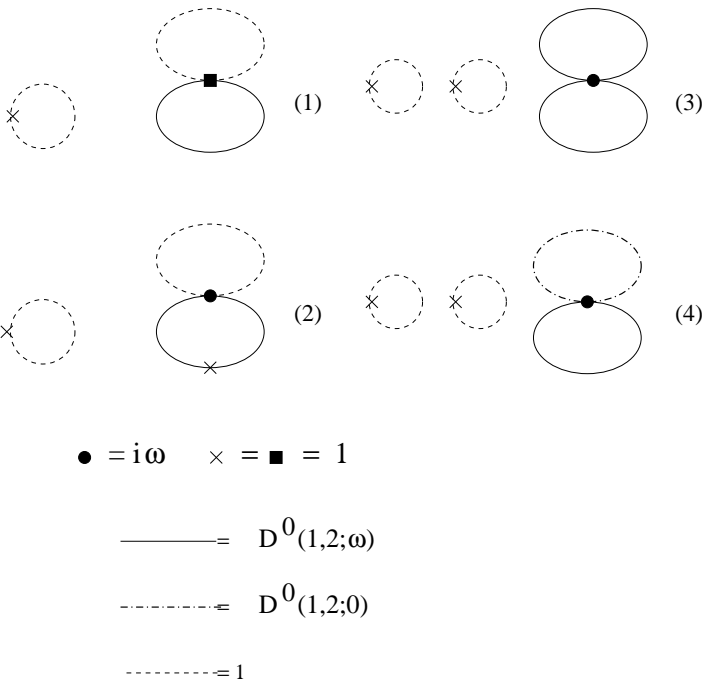

FIG. 4. Nonvanishing diagrams contribute to $R_{n p}(\omega)$ in the order of $(\Delta / \omega)^{3}$ for systems with broken time-reversal symmetry. The cross and the square, $\sim \operatorname{Str}\left[P^{2}\right]$ and $\sim \operatorname{Str}\left[P^{4}\right]$, respectively, come from the expansion in the prefactor, see Eq. (22). The dot, $\sim i \omega \operatorname{Str}\left[k P^{4}\right]$, comes from the effective interaction. The solid line stands for the bosonic propagator, the dashed line stands for the zero mode Grassmann propagator, and the dash-doted line stands for the nonzero mode Grassmann propagator. In the figure, we put $\Delta=1$.

$$
\begin{aligned}
\Delta R_{n p}^{\mathrm{u}}(\omega) & =\frac{1}{64} \operatorname{Re} \int D P e^{\frac{2 i \pi \omega}{\Delta}} e^{-\tilde{S}^{0}}\left[-8 \int d 1 \mathrm{~S} \operatorname{Tr}\left[P^{2}(1)\right] \int d 2 \mathrm{~S} \operatorname{Tr}\left[P^{4}(2)\right]-4 \int d 1 \mathrm{~S} \operatorname{Tr}\left[P^{2}(1)\right] \int d 2 \mathrm{~S} \operatorname{Tr}\left[P^{2}(2)\right] \tilde{S}_{\text {eff }}^{\text {int. }}\right] \\
\tilde{S}_{\text {eff }}^{\text {int. }} & =-\frac{\pi \nu}{2} i \omega \int d x_{\|} \mathrm{S} \operatorname{Tr}\left[k P^{4}\left(x_{\|}\right)\right] .
\end{aligned}
$$


To calculate Eq. (96), we need to separate the zero mode Grassmann fields from all the other fields and integrate them out first. Consequently we are left with a Gaussian integral involving the bosonic and nonzero mode Grassmann fields. Then we can factorize the integral into the product of the following pairs:

$$
\begin{aligned}
4 \pi \nu Z^{-1} \int D\left[a^{*} a b^{*} b \sigma^{*} \sigma \eta \eta^{*}\right] a_{k}^{*}(1) a_{k}(2) e^{-\tilde{S}^{0}}=\mathcal{D}^{0}(1,2 ;-\omega), \\
4 \pi \nu Z^{-1} \int D\left[a^{*} a b^{*} b \sigma^{*} \sigma \eta \eta^{*}\right] b_{k}^{*}(1) b_{k}(2) e^{-\tilde{S}^{0}}=\mathcal{D}^{0}(1,2 ; \omega), \\
4 \pi \nu Z^{-1} \int D\left[a^{*} a b^{*} b \sigma^{*} \sigma \eta \eta^{*}\right] \sigma_{k}^{*}(1) \sigma_{k}(2) e^{-\tilde{S}^{0}}=\mathcal{D}^{0}(1,2 ; 0), \\
4 \pi \nu Z^{-1} \int D\left[a^{*} a b^{*} b \sigma^{*} \sigma \eta \eta^{*}\right] \eta_{k}(1) \eta_{k}^{*}(2) e^{-\tilde{S}^{0}}=\mathcal{D}^{0}(1,2 ; 0),
\end{aligned}
$$

where $k=0,3$, and $\mathcal{D}^{0}(1,2 ; 0)$ satisfies the following equation:

$$
\hat{\mathcal{L}}_{R} \mathcal{D}^{0}(1,2 ; 0)=2 \pi[\delta(1-2)-1] .
$$

In the square integrable space, the overall factor, $Z$ can be approximated as

$$
Z=\int D\left[a^{*} a b^{*} b \sigma^{*} \sigma \eta \eta^{*}\right] e^{-\tilde{S}^{0}} \simeq \frac{\Delta^{2}}{(4 \pi)^{2}} \frac{1}{\omega^{2}} .
$$

We note that $Z \neq 1$ because $\tilde{S}^{0}$ is not supersymmtric and we do not integrate out zero mode Grassmann fields. Actually with nonzero modes integrated out, we come up with a factor $(1+a)$, where $a=\omega^{2} / \sum_{k} \lambda_{k}^{2}$ and the sum is over all the nonzero eigenvalues $\lambda_{k}$ of the PerronFrobenius operator ${ }^{10,30,48}$. In the universal region $\omega \ll$ $\lambda$, this factor crossovers to the universal value 1 . Furthermore, after integrating out zero mode bosonic fields, we obtain Eq. (102). The measure $D\left[a^{*} a b^{*} b \sigma^{*} \sigma \eta \eta^{*}\right]$ is on all the independent bosonic and nonzero mode Grassmann fields. Besides, one needs to take into account Eq. (14) to reduce the number of integration variables by half.

Eq. (96) involves the Gaussian integral of bosonic and nonzero mode Grassmann fields, which can reduced into the sum with each term the products of pairs [Eq. (97) to (100)]. Eq. (96) corresponds to 4 nonvanishing diagrams shown in Fig. 4. One should not confuse these diagrams with Fig. 1. Here the Hikami box in the 8-shaped diagram stands for the coupling between two diffusons, not a diffuson and a cooperon, as in the orthogonal case. It is important that such a diagram is exactly zero in the unitary case without the global transformation. In this way, the existence of these diagrams results from the supersymmetry breaking.

The first term in the r.h.s. of Eq. (96) gives

$$
\Delta R_{n p, 1}^{\mathrm{u}}(\omega)=-2 \frac{\Delta^{3}}{\pi^{3} \omega^{2}} \operatorname{Re}\left(e^{2 \pi i \omega / \Delta} \int d 3\left[\left\langle\mathcal{D}^{0}(3,3 ;-\omega)\right\rangle-\left\langle\mathcal{D}^{0}(3,3 ; \omega)\right\rangle\right]\right) \simeq-4 \frac{\Delta^{3}}{\pi^{3} \omega^{3}} \operatorname{Im} e^{2 \pi i \omega / \Delta}
$$

which is the contraction shown in Fig. 4(1). Above we use the fact that at $\omega \ll \lambda$, we need to take into account the zero mode only in the diffusons.

The second term in Eq. (96) has two nonvanishing contraction. One is shown in Fig. 4(2), while the other is shown in Fig. 4(3) and (4). In Fig. 4(2), only the bosonic fields are involved. By using Eqs. (97) and (98), we find it to be:

$$
\Delta R_{n p, 2}^{\mathrm{u}}(\omega)=-\frac{\Delta^{3}}{\pi^{3} \omega^{2}} \operatorname{Re}\left((-i \omega) e^{2 \pi i \omega / \Delta} \int d 2 \int d 3\left[\left\langle\mathcal{D}^{0}(3,2 ;-\omega) \mathcal{D}^{0}(2,3 ;-\omega)\right\rangle+\left\langle\mathcal{D}^{0}(3,2 ; \omega) \mathcal{D}^{0}(2,3 ; \omega)\right\rangle\right]\right) .
$$

It should be emphasized that the order of the arguments appearing in the product of two diffusons above is essentially different from what we calculated in Sec. IV B 1 [see Eq. (74)]. In fact, we can use Eq. (36) to reduce the two diffusons in Eq. (104) into one diffuson. Thus, we can approximate the product of two diffusons as $1 /( \pm i \omega)^{2}$ in the region $\omega \ll \lambda$.

Fig. 4(3) differs from Fig. 4(4) in that there the coupling occurs between bosonic fields. While Fig. 4(4), the coupling occurs between the bosonic and nonzero mode Grassmann fields. By using Eqs. (97) and (98), we find that Fig. 4(3) corresponds to

$$
\Delta R_{n p, 3}^{\mathrm{u}}(\omega)=-\frac{\Delta^{3}}{\pi^{3} \omega^{2}} \operatorname{Re}\left((-i \omega) e^{2 \pi i \omega / \Delta} \int d 3\left[\left\langle\mathcal{D}^{0}(3,3 ;-\omega) \mathcal{D}^{0}(3,3 ;-\omega)\right\rangle+\left\langle\mathcal{D}^{0}(3,3 ; \omega) \mathcal{D}^{0}(3,3 ; \omega)\right\rangle\right]\right) .
$$

By using Eqs. (97)-(100), we find that Fig. 4(4) corresponds to

$$
\Delta R_{n p, 4}^{\mathrm{u}}(\omega)=\frac{\Delta^{3}}{\pi^{3} \omega^{2}} \operatorname{Re}\left((-i \omega) e^{2 \pi i \omega / \Delta} \int d 3\left[\left\langle\mathcal{D}^{0}(3,3 ;-\omega) \mathcal{D}^{0}(3,3 ; 0)\right\rangle+\left\langle\mathcal{D}^{0}(3,3 ; \omega) \mathcal{D}^{0}(3,3 ; 0)\right\rangle\right]\right)
$$


In the disordered case, one is easy to check that $\Delta R_{n p}^{\mathrm{u}}(\omega)$ vanishes using the zero mode diffuson: $\left\langle\mathcal{D}^{0}(1,2 ; \omega)\right\rangle=1 /-i \omega^{+}$and $\left\langle\mathcal{D}^{0}(1,2 ; 0)\right\rangle=0$. However, it is not so in the crossover region $\Delta \ll \omega \sim t_{E}^{-1} \ll t_{\text {erg }}^{-1}$. Instead, as we see below, Eqs. (103)-(106) lead to the Ehrenfest oscillation with the period $\left(\Delta^{-1}+\alpha t_{E}\right)^{-1}$ with $\alpha$ a universal numerical factor.

\section{The Ehrenfest oscillation in $R_{n p}^{\mathrm{u}}(\omega)$}

Now we show that in the crossover region, Eqs. (103)(106) lead to the Ehrenfest oscillation, but with different dependence of the period on $t_{E}$ compared to the perturbative part.

First, according to Eq. (75), we can rewrite Eq. (105) as

$$
\Delta R_{n p, 3}^{\mathrm{u}}(\omega)=\frac{\Delta^{3}}{\pi^{3} \omega^{2}} \operatorname{Re}\left[e^{2 \pi i \omega / \Delta} \frac{\Gamma_{2}^{2}(\omega)+\Gamma_{2}^{2}(-\omega)}{-i \omega}\right] .
$$

In the case of $\lambda_{2} \omega^{2} / \lambda^{3} \ll 1$, Eq. (107) is simplified as

$$
\Delta R_{n p, 3}^{\mathrm{u}}(\omega)=-2 \frac{\Delta^{3}}{\pi^{3} \omega^{2}} \sin \frac{2 \pi \omega}{\Delta} \cos 2 \omega t_{E}
$$

To calculate Eq. (106), first we understand $\mathcal{D}^{0}(3,3 ; 0)$ in the way of $\mathcal{D}^{0}(3,3 ; 0)=$ $\lim _{\omega_{1} \rightarrow 0}\left[\mathcal{D}^{0}\left(3,3 ; \omega_{1}\right)+\mathcal{D}^{0}\left(3,3 ;-\omega_{1}\right)\right] / 2$ where $\mathcal{D}^{0}\left(1,2 ; \omega_{1}\right)$ should be considered as the solution of the equation below:

$$
\begin{aligned}
& \left(-i \omega_{1}^{+}+\hat{\mathcal{L}}_{R}\right) \mathcal{D}^{0}\left(1,2 ; \omega_{1}\right)=2 \pi[\delta(1-2)-1] . \quad(109) \quad \frac{\begin{array}{c}
\text { Taking into account Eqs. (86) and (9), we can formally } \\
\text { express } R(\omega) \text { as the following : }
\end{array}}{R(\omega)=1+a+\sum_{n}\left(\frac{\Delta}{\omega}\right)^{n} C_{n}\left(\omega t_{E}\right)+1\left[\cos \frac{2 \pi \omega}{\Delta} \sum_{n}\left(\frac{\Delta}{\omega}\right)^{n} D_{n}\left(\omega t_{E}\right)+\sin \frac{2 \pi \omega}{\Delta} \sum_{n}\left(\frac{\Delta}{\omega}\right)^{n} E_{n}\left(\omega t_{E}\right)\right] .}(113)
\end{aligned}
$$

Then following the procedure of deriving Eq. (74), we find that $\left\langle\mathcal{D}^{0}(3,3 ; \omega) \mathcal{D}^{0}\left(3,3 ; \omega_{1}\right)\right\rangle=-\Gamma_{2}^{2}\left(\frac{\omega+\omega_{1}}{2}\right) / \omega^{+} \omega_{1}^{+}$. Thus we obtain:

$$
\left\langle\mathcal{D}^{0}(3,3 ; \omega) \mathcal{D}^{0}(3,3 ; 0)\right\rangle=-\frac{1}{\omega} \frac{\partial}{\partial \omega} \Gamma_{2}^{2}\left(\frac{\omega}{2}\right) .
$$

Substituting Eq. (110) into Eq. (106), we find that $\Delta R_{n p, 4}^{\mathrm{u}}(\omega)$ is

$$
\Delta R_{n p, 4}^{\mathrm{u}}(\omega)=-2 \frac{\Delta^{3}}{\pi^{3} \omega^{3}} \sin \frac{2 \pi \omega}{\Delta} \omega \operatorname{Re} \frac{\partial}{\partial \omega} \Gamma_{2}^{2}\left(\frac{\omega}{2}\right) .
$$

In the case of $\lambda_{2} \omega^{2} / \lambda^{3} \ll 1$, Eq. (111) is simplified as

$$
\Delta R_{n p, 4}^{\mathrm{u}}(\omega)=2 \frac{\Delta^{3}}{\pi^{3} \omega^{3}} \sin \frac{2 \pi \omega}{\Delta} \omega t_{E} \sin \omega t_{E} .
$$

Collecting Eqs. (103), (104), (107) and (111) together, we find that the leading quantum corrections in the nonperturbative part of $R(\omega)$ is Eq. (9). Thereby $R_{n p}^{\mathrm{u}}(\omega)$ acquires an oscillation correction with the period $\left(\Delta^{-1}+\alpha t_{E}\right)^{-1}$ with $\alpha$ a universal numerical factor. Actually we can find the similar results for systems with time-reversal symmetry, but the amplitudes are much smaller, which are proportional to $(\Delta / \omega)^{5} \cos (2 \pi \omega / \Delta)$ or $(\Delta / \omega)^{5} \sin (2 \pi \omega / \Delta)$.

In the low $\omega$ limit: $\omega t_{E} \ll 1, \Delta R_{n p}^{\mathrm{u}}(\omega) \sim \Delta^{3} t_{E}^{2} / \omega$. It is a small quantum correction to the universal WignerDyson statistics. However, we are not able to find this term by using the method developed in Ref. 10 . In the large $\omega$ limit: $\omega t_{E} \gg 1$, we see that the universal Wigner-Dyson statistics acquires a small universal correction $\sim(\Delta / \omega)^{3} e^{2 \pi i \omega / \Delta}$. But it is smaller than the nonuniversal correction, which is $\sim(\Delta / \lambda)^{2} e^{2 \pi i \omega / \Delta}$.
The factor $a \sim \omega^{2} / \lambda^{2}$ arises from making the Gaussian approximation to the nonzero modes of the PerronFrobenius operator ${ }^{30,48}$. We do not study this term in this paper because it does not lead to the Ehrenfest oscillations. For disordered systems, a similar term, $\sim \omega^{2} / E_{T h}^{2}$ was found in Ref. 10. In general, $C_{n}, D_{n}$ and $E_{n}$ are oscillating functions of $\omega t_{E}$ and proportional to $\Gamma_{n}(\omega)$. In the disordered limit, $C_{n}, D_{n}$ and $E_{n}$ become universal constants.

\section{CONCLUSIONS}

In this paper, we study the electron energy statistics in chaotic quantum dots with one macroscopic size $L$. For such systems, the inverse ergodic time and the Lyapunov exponent are of the same order: $t_{\text {erg }}^{-1} \sim \lambda \sim \frac{v_{F}}{L}$. Consequently, in the semiclassical limit, the Ehrenfest time $t_{E}$ opens an intermediate region $\Delta \ll t_{E}^{-1} \sim \omega \ll t_{\text {erg }}^{-1}$. We study the behavior of the two level correlation function, $R(\omega)$ in the universal region $\Delta \ll \omega \ll t_{\text {erg }}^{-1}$ in systems with broken time-reversal symmetry. Surprisingly, it is found that $R(\omega)$ deviates from the universal WignerDyson statistics. Basically, we find that $R(\omega)$ acquires two types of oscillation corrections in the crossover region $t_{E}^{-1} \sim \omega \ll t_{\text {erg }}^{-1}$. The oscillation periods have different dependence on $t_{E}$. The Ehrenfest oscillations in the perturbative part have the period proportional to $t_{E}^{-1}$, while the Ehrenfest oscillations in the nonperturbative part have the period $\left(\Delta^{-1}+\alpha t_{E}\right)^{-1}$ with $\alpha$ being some universal numerical factor. These additional Ehrenfest oscillations are small corrections to the universal RMT 
results. In particular, for $R_{p}^{\mathrm{u}}(\omega)$ described by Eq. (28), the exact truncation at the term $\sim \omega^{-2}$ does not imply the disappearance of quantum corrections. Instead, it is due to the cancellations of contributions arising from Hikami boxes associating with different kinds of vertices at the limit $\omega t_{E} \rightarrow 0$. The Ehrenfest oscillations are just the reminiscence of such cancellations.

In this paper, only the leading Ehrenfest oscillation corrections are calculated. Actually there is a general expression, Eq. (113) in the region $\Delta \ll \omega \ll t_{\text {erg }}^{-1}$. The functions $\tilde{C}_{n}(x)$ [hence $C_{n}(x)$ ], $D_{n}(x)$ and $E_{n}(x)$ are oscillating at $\omega t_{E} \gtrsim 1$. In the limit $n \omega t_{E} \ll 1, \tilde{C}_{n}$ (hence $\left.C_{n}\right), D_{n}$ and $E_{n}$ crossover to their universal values. In particular, in the GUE case, $C_{2}=-D_{2}=-1 / 2$, and all other coefficients vanish. The universal values of $\tilde{C}_{n}(x)$ [hence $C_{n}(x)$ ], $D_{n}(x)$ and $E_{n}(x)$ do not depend on the magnitude of the regularizer [the last term in Eq. (12)]. But it depends on the order of how we take the limits: $\omega \rightarrow 0$ and $\tau_{q} \rightarrow \infty$. Actually we take the limit $\omega \rightarrow 0$ before $\tau_{q} \rightarrow \infty$. In contrast, the boundary of the region where the universality exists $\left(\omega \ll t_{E}^{-1}\right)$ does logarithmically depend on the magnitude of $\tau_{q}$. The regularizer describe the coupling between classical trajectories. In this sense, it plays a role similar to that played by the interaction between particles in nonideal Bose gases. There the scaling theory describes the universal behavior at the transition point and the critical indices do not depend on interactions. However, the region for the scaling to be applicable does depend on the interaction. While in ideal gases, the scaling is absent. It is important that the results presented here hold only at $\Delta \ll \omega \ll \lambda$, where the saddle point approximation to Eqs. (10) and (12) is possible.

We note that $\Gamma_{n}(\omega)$ is not analytical at $1 / \tau_{q}$ in the large $\omega$ limit $t_{E}^{-1} \ll \omega<\lambda$. Actually following from Eqs. (4) and (82), we find that

$$
\Gamma_{n}(\omega) \sim\left(\frac{1}{\tau_{q}}\right)^{\omega^{2} /\left(\lambda^{3} / \lambda_{2}\right)+i \omega / \lambda}, \quad t_{E}^{-1} \ll \omega<\lambda .
$$

From this, we see that corrections to $R(\omega)$, predicted by the Gutzwiller formula, are proportional to $\tau_{q}^{-\alpha}, 0<$ $\alpha \ll 1$. In this way, any attempts of establishing a $1 / \tau_{q}$ expansion are prohibited.

At higher $\omega, \omega \gtrsim \lambda \sim t_{\text {erg }}^{-1}$, the nonzero mode contributions turn out to be important. In this case, the saddle point approximation is no longer applicable. A refined technique is desired to study the oscillation, claimed in Ref. 48 for generic chaotic quantum dots.

In this paper, we consider chaotic quantum dots where $\lambda \sim t_{\text {erg }}^{-1} \sim \frac{v_{F}}{L}$. Actually, it is possible that $\lambda \gg t_{\text {erg }}^{-1}$. In the latter case, the dot has large enough size to contain a lot of classical impurities inside. In this case, the Lyapunov exponent $\lambda \sim \operatorname{nav}_{F}$, where $n$ is the concentration of impurities and $a$ is the size of classical impurities ${ }^{49}$. The ergodic time (Thouless time) $t_{\text {erg }}=L^{2} / D \gg \lambda^{-1}$. Here $D$ is the classical diffusion constant. In this case, contributions from nonzero modes are possible to be described by the saddle point approximation. The similar Ehrenfest oscillations are expected to exist at $t_{\text {erg }}^{-1} \lesssim \omega \sim$ $t_{E}^{-1}<\lambda$. We leave this work in the future.

Based on the present work, it is unclear whether the BGS conjecture ${ }^{13}$ may work in the universal region $\omega \ll$ $t_{\text {erg }}^{-1}$ for generic quantum chaotic systems. In other words, are the fluctuations of energy levels described by the universal Wigner-Dyson statistics in such systems? According to Eq. (113), we point out that in chaotic quantum billiards a new scale, the Ehrenfest time appears, which results in the new types of oscillations at $\omega \sim t_{E}^{-1}$. These oscillations are not expected by either the RMT, zero mode nonlinear supermatrix $\sigma$ model or the Gutzwiller trace formula. Secondly, despite of the appearance of the Ehrenfest oscillations in the region $\omega \sim t_{E}^{-1} \gg \Delta$, their amplitudes are small. In this way, $R(\omega)$ is still dominated by the universal Wigner-Dyson statistics. Finally, the behavior of $R(\omega)$ at $\omega \sim \Delta$ remains an open problem. Indeed, in the present work we use the saddle point approximation to take into account the zero mode contributions. Furthermore, the perturbation theory near the saddle points predicts the regions for the terms in the expansion Eq. (113) to approach their universal limits. However, we are not aware of the limit of the sum of such an asymptotic expansion.

\section{ACKNOWLEDGEMENTS}

We thank I. L. Aleiner, K. B. Efetov, A. Kamenev, B. I. Shklovskii and M. G. Vavilov for a lot of fruitful discussions. The work is supported by NSF grant No. 0120702 .

\section{APPENDIX A: THE DERIVATION OF EQUATION (79)}

In this Appendix we prove Eq. (79). We will follow the general method developed in Ref. 22. In the discussions below, we ignore the small regularizer in Eq. (78) for the moment. Moreover, we turn to the time representation. Then employing the change of variables

$$
\begin{aligned}
& z=\left(z_{1}, z_{2}\right), \quad \alpha=\left(\alpha_{1}, \alpha_{2}\right), \\
& z_{1}=\ln \left[\theta_{1}^{2}+\left(\frac{\rho_{1}}{L}\right)^{2}\right]^{1 / 2}, \quad \alpha_{1}=\arctan \frac{\theta_{1} L}{\rho_{1}}, \\
& z_{2}=\ln \left[\theta_{2}^{2}+\left(\frac{\rho_{2}}{L}\right)^{2}\right]^{1 / 2}, \quad \alpha_{2}=\arctan \frac{\theta_{2} L}{\rho_{2}},
\end{aligned}
$$

we rewrite Eq. (78) as (after averaging over the coordinates of the center of mass)

$$
\left(\frac{\partial}{\partial t}+\hat{D}_{1}+\hat{D}_{2}\right) N(z, \alpha ; 2,2)=0
$$




$$
\begin{aligned}
& \hat{D}_{1}=-B_{1}(t) \sin 2 \alpha_{1} \frac{\partial}{\partial z_{1}}+\left[B_{1}(t) \cos 2 \alpha_{1}+B_{2}(t)\right] \frac{\partial}{\partial \alpha_{1}}, \\
& \hat{D}_{2}=-B_{1}(t) \sin 2 \alpha_{2} \frac{\partial}{\partial z_{2}}+\left[B_{1}(t) \cos 2 \alpha_{2}+B_{2}(t)\right] \frac{\partial}{\partial \alpha_{2}}, \\
& B_{1,2}(t)=\left.\frac{v_{F}}{2 L} \mp \frac{L}{2 p_{F}} \frac{\partial^{2} U}{\partial R_{\perp}^{2}}\right|_{\mathbf{R}=\mathbf{R}\left(t, \mathbf{R}_{0}\right)} .
\end{aligned}
$$

Introducing the change of variables

$$
x=\frac{z_{1}+z_{2}}{2}, \quad y=z_{1}-z_{2},
$$

we obtain:

$\hat{D}_{1}+\hat{D}_{2}=$ $-\frac{1}{2} B_{1}(t)\left(\sin 2 \alpha_{1}+\sin 2 \alpha_{2}\right) \frac{\partial}{\partial x}+B_{1}(t)\left(\sin 2 \alpha_{1}-\sin 2 \alpha_{2}\right) \frac{\partial}{\partial y} \int_{0}^{t} d t B_{1}(t) \sin \left[2 \hat{\alpha}_{1}\left(\alpha_{1}^{0}, t\right)\right]=\int_{0}^{t} d t B_{1}(t) \sin \left[2 \hat{\alpha}_{2}\left(\alpha_{2}^{0}, t\right)\right]$. $+\left[B_{1}(t) \cos 2 \alpha_{1}+B_{2}(t)\right] \frac{\partial}{\partial \alpha_{1}}+\left[B_{1}(t) \cos 2 \alpha_{2}+B_{2}(t)\right] \frac{\partial}{\partial \alpha_{2}}$.

The formal solution of Eq. (A2) is

$$
\begin{aligned}
& N(t ; x, y ; 2,2)= \\
& \exp \left[C_{1}\left(t, \alpha_{1,2}\right) \frac{\partial}{\partial x}\right] N\left(0 ; x, y, \hat{\alpha}^{0}(\alpha, t) ; 2,2\right),
\end{aligned}
$$

where $C_{1}$ is

$$
C_{1}\left(t, \alpha_{1,2}\right)=\frac{1}{2} \int_{0}^{t} d t_{1} B_{1}\left(t_{1}\right)\left(\sin 2 \hat{\alpha}_{1}+\sin 2 \hat{\alpha}_{2}\right)
$$

The functions of $y(t)$ and $\hat{\alpha}_{1,2}\left(\alpha_{1,2}^{0}, t\right)$ satisfy the following equations:

$$
\begin{aligned}
& \frac{\partial y}{\partial t}=B_{1}(t)\left(\sin 2 \alpha_{1}-\sin 2 \alpha_{2}\right), \\
& \frac{\partial}{\partial t} \hat{\alpha}_{1,2}=B_{1}(t) \cos 2 \hat{\alpha}_{1,2}+B_{2}(t), \\
& \hat{\alpha}_{1,2}\left(\alpha_{1,2}^{0}, 0\right)=\alpha_{1,2}^{0},
\end{aligned}
$$

with $\hat{\alpha}_{1,2}$ implicitly defined by

$$
\hat{\alpha}_{1,2}\left[\hat{\alpha}_{1,2}^{0}\left(\alpha_{1,2}, t\right), t\right]=\hat{\alpha}_{1,2} .
$$

The formal solution of $y(t)$ is

$$
y(t)=y(0)+\int_{0}^{t} d t_{1} B_{1}\left(t_{1}\right)\left(\sin 2 \alpha_{1}-\sin 2 \alpha_{2}\right)
$$

with $y(0)$ being the initial condition.

We are interested in the region $t \gtrsim \lambda^{-1}$. In this case, $\hat{\alpha}_{1,2}\left(\hat{\alpha}_{1,2}^{0}, t\right)$ become self-averaging over the coordinates of the center of mass and no longer depend on the initial conditions. Consequently, at such large times, $y(t)$ reaches a constant depending on the initial value [see Eq. (A10)]. In this way, $N(t ; x, y ; 2,2)$ depends on $y$ parametrically and has no $\alpha_{1,2}$ dependence. The evolution of $N(t ; x, y ; 2,2)$ is governed by the Fokker-Planck type equation:

$$
\left[\frac{\partial}{\partial t}-\mathcal{F}\left(\frac{\partial}{\partial x}\right)\right] N(t ; x, y ; 2,2)=0,
$$

where $\mathcal{F}\left(\frac{\partial}{\partial x}\right)$ is defined as

$$
\mathcal{F}\left(\frac{\partial}{\partial x}\right)=\lim _{t \rightarrow \infty} \frac{1}{t} \ln \int \frac{d \mathbf{n} d \mathbf{R}}{2 \pi A} \exp \left[B(t)\left(\frac{\partial}{\partial x}\right)\right] .
$$

Here we keep in mind the important fact that $\int_{0}^{t} d t B_{1}(t) \sin \left[2 \hat{\alpha}_{1,2}\left(\alpha_{1,2}^{0}, t\right)\right]$ does not depend on $\alpha_{1,2}$ In this way, $\mathcal{F}$ has no $\frac{\partial}{\partial y}$ dependence.

$$
B(t)=\int_{0}^{t} d t B_{1}(t) \sin \left[2 \hat{\alpha}\left(\alpha^{0}, t\right)\right],
$$

where $\alpha$ can take the subscript either 1 or 2 .

Because $N(t ; x, y ; 2,2)$ slowly varies over $x$, we expand $\mathcal{F}$ up to the second order in $\frac{\partial}{\partial x}$ to get:

$$
\mathcal{F}\left(\frac{\partial}{\partial x}\right)=\lambda \frac{\partial}{\partial x}+\frac{\lambda_{2}}{2} \frac{\partial^{2}}{\partial x^{2}},
$$

where

$$
\lambda=\lim _{t \rightarrow \infty} \frac{1}{t} \int \frac{d \mathbf{n} d \mathbf{R}}{2 \pi A} B(t)
$$

and

$$
\lambda_{2}=\lim _{t \rightarrow \infty} \frac{1}{t}\left\{\left[\int \frac{d \mathbf{n} d \mathbf{R}}{2 \pi A} B^{2}(t)\right]-\lambda^{2} t^{2}\right\} .
$$

It is important that here $\lambda$ and $\lambda_{2}$ are exactly the same as those appearing in $\Gamma_{2}(\omega)$ because $B(t)$ is the same as that in Ref. 22. This is not surprising because mathematically, $\lambda$ is determined by the eigenvalue of the stability matrix of a trajectory ${ }^{50}$. Returning to the frequency representation, we find Eq. (79).

${ }^{1}$ See, for examples, H.U. Baranger, in Nanotechnology, edited by G. Timp (American Institute of Physics, New York, 1998); Y. Alhassid, Rev. Mod. Phys. 72, 895 (2000).

${ }^{2}$ E. P. Wigner, Ann. Math. 53, 36 (1951).

${ }^{3}$ C. E. Porter (editor) Statistical Theory of Spectra: Fluctuations, (Academic Press, New York, 1965).

${ }^{4}$ L. P. Gor'kov and G. M. Eliashberg, Zh. Eksp. Teor. Fiz. 48, 1407 (1965) [Sov. Phys. JETP 21, 940 (1965)]. 
${ }^{5}$ K. B. Efetov, Supersymmetry in Disorder and Chaos, (Cambridge University Press, UK, 1997); Adv. Phys. 32, 53 (1983).

${ }^{6}$ B. L. Altshuler and B. I. Shklovskii, Zh. Eksp. Teor. Fiz. 91, 220 (1986) [Sov. Phys. JETP 64, 127 (1986)].

${ }^{7}$ M. C. Gutzwiller, Chaos in Classical and Quantum Mechanics, (Springer, New York, 1990).

8 M. L. Mehta, Random Matrices, (Academic Press, New York, 1990).

${ }^{9}$ F. J. Dyson, J. Math. Phys. 3, 140, 157, 166 (1962).

${ }^{10}$ V. E. Kravtsov and A. D. Mirlin, Zh. Eksp. Teor. Fiz. 60, 645 (1994) [JETP Lett. 60, 656 (1994)].

${ }^{11}$ R. A. Smith, I. V. Lerner, and B. L. Altshuler, Phys. Rev. B 58,10343 (1998).

${ }^{12}$ K. B. Efetov, A. I. Larkin, and D. E. Khmelnitskii, Zh. Eksp. Teor. Fiz. 79, 1120 (1980) [Sov. Phys. JETP 52, 568 (1980)]; F. J. Wegner, Z. Phys. B 35, 207 (1979).

13 O. Bohigas, M. -J. Giannoni, and C. Schmit, Phys. Rev. Lett. 52, 1 (1984).

${ }^{14}$ M. C. Gutzwiller, J. Math. Phys. 12, 343 (1971).

${ }^{15}$ M. V. Berry, Proc. Roy. Soc. London A 400, 229 (1985).

16 J. H. Hannay and A. M. Ozorio de Almeida, J. Phys. A 17, 3429 (1984).

${ }^{17}$ E. B. Bogomolny and J. P. Keating, Phys. Rev. Lett. 77, 1472 (1996); J. P. Keating, in Supersymmetry and Trace Formulae, edited by I. V. Lerner, J. P. Keating, and D. E. Khmelnitskii, NATO series B (Kluwer Academic Press, Dordrecht, 1999).

18 A. I. Larkin and Yu. N. Ovchinnikov, Zh. Eksp. Teor. Fiz. 55, 2262 (1968) [Sov. Phys. JETP 28, 1200 (1969)].

${ }^{19}$ G. P. Berman and G. M. Zaslavsky, Dokl. Akad. Nauk USSR 240, 1081 (1978); Physica A 91, 450 (1978). See also the review: G. M. Zaslavsky, Phys. Rep. 80, 157 (1981); F. M. Izrailev, Phys. Rep. 196, 299 (1990).

${ }^{20}$ M. Wilkinson, J. Phys. A 20, 2415 (1987).

${ }^{21}$ N. Argaman, Phys. Rev. Lett. 75 2750, (1995); Phys. Rev. B 53, 7035 (1996).

${ }^{22}$ I. L. Aleiner and A. I. Larkin, Phys. Rev. B 54, 14423 (1996).

${ }^{23}$ S. Hikami, Phys. Rev. B 24, 2671 (1981).

${ }^{24}$ O. Agam, I. L. Aleiner, and A. I. Larkin, Phys. Rev. Lett. 85, 3153 (2000).

25 J. Tworzydlo, A. Tajic, H. Schomerus, and C. W. J. Beenakker, Phys. Rev. B 68, 115313 (2003).

${ }^{26}$ M. G. Vavilov and A. I. Larkin, Phys. Rev. B 67, 115335(2003)

${ }^{27} \mathrm{Ph}$. Jacquod, H. Schomerus, and C. W. J. Beenakker, Phys. Rev. Lett. 90, 207004 (2003).

${ }^{28}$ I. L. Aleiner and A. I. Larkin, Phys. Rev. E 55, R1243 (1997).

${ }^{29}$ We notice that in Ref. 28 , it is a mistake to put $I_{1}+I_{2}$ [see Eqs. (37) and (38)] to be zero. Consequently, the re- sult there is in a slight difference from what we show in Eq. (3) and can not be expressed as the second derivative of the free energy $F_{2}(\omega)$.

${ }^{30}$ A. V. Andreev, B. D. Simons, O. Agam, and B. L. Altshuler, Nucl. Phys. B 482, 536 (1996); B. D. Simons, O. Agam, and A. V. Andreev, J. Math. Phys. 38, 1982 (1997).

${ }^{31}$ B. A. Muzykantskii and D. E. Khmelnitskii, Zh. Eksp. Teor. Fiz. 62, 68 (1995) [JETP Lett. 62, 76 (1995)].

${ }^{32}$ V. R. Kogan and K. B. Efetov, Phys. Rev. B 67, 245312 (2003).

33 A. V. Andreev and B. L. Altshuler, Phys. Rev. Lett. 75, 902 (1995); J. Math. Phys. 37, 4968 (1996).

34 A. Altland, C. R. Offer, and B. D. Simons, in Supersymmetry and Trace Formulae, edited by I. V. Lerner, J. P. Keating, and D. E. Khmelnitskii, NATO series B (Kluwer Academic Press, Dordrecht, 1999).

${ }^{35}$ K. B. Efetov and A. I. Larkin, in preparation.

${ }^{36}$ J. B. Keller, J. Opt. Soc. Am. 52, 116 (1962).

${ }^{37}$ G. Vattay, A. Wirzba, and P. E. Rosenqvist, Phys. Rev. Lett. 73, 2304 (1994).

${ }^{38}$ H. Parimack, H. Schanz, U. Smilansky, and I. Ussishkin, Phys. Rev. Lett. 76, 1615 (1996); J. Phys. A 30, 6693 (1997); R. S. Whitney, I. V. Lerner, and R. A. Smith, Wave in Random Media 9, 179 (1999).

39 D. Ruelle, Phys. Rev. Lett. 56, 405 (1986); M. Pollicot, Ann. Math. 131, 331 (1990).

40 See, for example, P. Gaspard, Phys. Rev. E 53, 4379 (1996) and references therein.

${ }^{41}$ C. Beck and F. Schlogl, Thermodynamics of Chaotic Systems, (Cambridge University Press, Cambridge, UK, 1993).

42 A. Kamenev and M. Mezard, J. Phys. A 32, 4373 (1999), Phys. Rev. B 60, 3944 (1999); I. V. Yurkevich and I. V. Lerner, Phys. Rev. B 60, 3955 (1999).

${ }^{43}$ In the language of quantum disorders, the diffuson stands for the interference between a path and itself, and the cooperon stands for the interference between a path and its time-reversed partner.

${ }^{44}$ M. Sieber and K. Richter, Physica Scripta T90, 128 (2001).

45 A. A. Abrikosov, L. P. Gor'kov, and Ye. I. Dzyaloshinskii, Quantum Field Theoretical Methods in Statistical Physics (Pergamon Press, New York, 1969).

${ }^{46}$ I. V. Gornyi and A. D. Mirlin, J. Low. Temp. Phys. 126, 1339 (2002).

${ }^{47}$ S. Willard, Basic Topology, (McGraw-Hill, Berkshire, England, 1979).

${ }^{48}$ Ya. Blanter, A. D. Mirlin, and B. A. Muzykantskii, Phys. Rev. B 63, 235315 (2001).

${ }^{49}$ H. van Beijeren and J. R. Dorfman, Phys. Rev. Lett. 74, 4412 (1995).

${ }^{50}$ A. J. Lichtenberg and M. A. Lieberman, Regular and Chaotic Dynamics, 2nd ed. (Springer-Verlag, Birlin, 1992). 